\title{
Optimal Control of Investment-Reinsurance Problem for an Insurer with Jump-Diffusion Risk Process: Independence of Brownian Motions
}

\author{
De-Lei Sheng, ${ }^{1}$ Ximin Rong, ${ }^{1,2}$ and Hui Zhao ${ }^{1}$ \\ ${ }^{1}$ Department of Mathematics, Tianjin University, Tianjin 300072, China \\ ${ }^{2}$ Center for Applied Mathematics, Tianjin University, Tianjin 300072, China \\ Correspondence should be addressed to De-Lei Sheng; tjhbsdl@126.com
}

Received 18 March 2014; Revised 20 June 2014; Accepted 25 June 2014; Published 24 July 2014

Academic Editor: Simone Marsiglio

Copyright (C) 2014 De-Lei Sheng et al. This is an open access article distributed under the Creative Commons Attribution License, which permits unrestricted use, distribution, and reproduction in any medium, provided the original work is properly cited.

\begin{abstract}
This paper investigates the excess-of-loss reinsurance and investment problem for a compound Poisson jump-diffusion risk process, with the risk asset price modeled by a constant elasticity of variance (CEV) model. It aims at obtaining the explicit optimal control strategy and the optimal value function. Applying stochastic control technique of jump diffusion, a Hamilton-Jacobi-Bellman (HJB) equation is established. Moreover, we show that a closed-form solution for the HJB equation can be found by maximizing the insurer's exponential utility of terminal wealth with the independence of two Brownian motions $W(t)$ and $W_{1}(t)$. A verification theorem is also proved to verify that the solution of HJB equation is indeed a solution of this optimal control problem. Then, we quantitatively analyze the effect of different parameter impacts on optimal control strategy and the optimal value function, which show that optimal control strategy is decreasing with the initial wealth $x$ and decreasing with the volatility rate of risk asset price. However, the optimal value function $V(t ; x ; s)$ is increasing with the appreciation rate $\mu$ of risk asset.
\end{abstract}

\section{Introduction}

By means of investment and reinsurance, insurers can protect themselves against potentially large losses or ensure their earnings remain relatively stable. Therefore, many optimal investment and reinsurance problems have arisen in insurance risk management and have been extensively studied in the literature.

In the older forms, reinsurance was often referred to as "proportional" reinsurance; few studies pay attention to reinsurance. Since Borch [1] studied the safety loading of reinsurance premiums, a vast amount of literature is particularly concerned about reinsurance. However, the excess-of-loss reinsurance is a tool commonly employed in risk management in the recent thirty years. Tapiero and Zuckerman [2] gave the optimum excess-loss reinsurance under a dynamic framework. Taylor [3] studied reserving consecutive layers of inwards excess-of-loss reinsurance. Cao and $\mathrm{Xu}[4]$ investigated both proportional and excess-of-loss reinsurance under investment gains. Gu et al. [5] investigated optimal control of excess-of-loss reinsurance and investment for insurers under a constant elasticity of variance model but without compound Poisson jump in their research. Zhao et al. [6] studied optimal excess-of-loss reinsurance and investment problem for an insurer with jump-diffusion risk process under the Heston model.

It is well known that the compound Poisson process is the most popular and useful model to describe claims process ever since the classical Cramér-Lundberg model in risk theory. However, as a compound Poisson process perturbed by a standard Brownian motion, jump diffusion has been researched extensively in the recent ten years. Jump diffusion can give more practical description of claims than continuous models, which widely used to describe dynamics of surplus process. Yang and Zhang [7] investigated the problem of optimal investment for insurer with jump-diffusion risk process. Li et al. [8] studied the threshold dividend strategy for renewal jump-diffusion process. Ruan et al. [9] studied the optimal portfolio and consumption with habit formation in a jump-diffusion market. 
Not only does the insurer cede part of premiums for reinsurance, but the insurer also invests in a financial market consisting of one risk-free asset and a risk asset. The constant elasticity of variance (CEV) model describes the volatility of risk asset that received widespread interests after being proposed by Cox and Ross [10] for European option pricing, partly because the CEV model can capture the implied volatility skew and can explain the volatility smile as well. Hsu et al. [11] gave the integration and detailed derivation for constant elasticity of variance (CEV) option pricing model. Campi et al. [12] investigated systematic equitybased credit risk for a CEV model with jump to default. Applying Legendre transform and dual theory, Xiao et al. [13] investigated analytical strategies for annuity contracts under constant elasticity of variance model. The CEV model was applied to the optimal investment and reinsurance problems in $\mathrm{Gu}$ et al. [14]. By maximizing the expected exponential utility of terminal wealth, Lin and Li [15] studied the optimal reinsurance and investment for a jump-diffusion risk process under the CEV model.

The goal of this paper is therefore to investigate the optimal control strategy of excess-of-loss reinsurance and investment problem for an insurer with compound Poisson jump-diffusion risk process from the stochastic control point of view. Under the hypothesis that two Brownian motions $W(t)$ and $W_{1}(t)$ are mutually independent, we present three main results. We first give the optimal excess-of-loss reinsurance strategy and investment strategy, respectively, according to the first-order necessary condition of maximum. We also obtain the optimal value function by plugging a reasonable conjecture into the $\mathrm{HJB}$ equation and solving linear second-order partial differential equations. In the third part, we specialize in a verification theorem. We also prove the verification theorem in detail inspired by the results of Taksar and Zeng [16], Gu et al. [5], and Zhao et al. [6]. At last, we present some numerical examples to illustrate the impacts of model parameters.

The paper is organized as follows. Section 2 introduces the risk asset price model under the CEV model and the wealth process with jump diffusion. In Section 3, we consider the optimal excess-of-loss reinsurance and investment strategy and a verification theorem as well. Section 4 contains some numerical simulations and theoretical results. Section 5 brings this paper to an end.

\section{The Model}

Throughout this paper, $\left(\Omega, \mathscr{F}, P,\left\{\mathscr{F}_{t}\right\}_{0 \leq t \leq T}\right)$ denotes a complete probability space satisfying the usual condition, where a finite constant $T>0$ represents the investment time horizon; two standard Brownian motions $W_{1}(t)$ and $W(t)$ are independent of each other; $\gamma$ is a Poisson random measure; $\mathscr{F}_{t}:=\mathscr{F}_{t}^{W} \vee \mathscr{F}_{t}^{W_{1}} \vee \mathscr{F}_{t}^{\gamma}$ represents the minimal $\sigma$-field generated by $\mathscr{F}_{t}^{W}, \mathscr{F}_{t}^{W_{1}}$, and $\mathscr{F}_{t}^{\gamma}$, which contains all the information available until time $t$. All stochastic processes involved in this paper are supposed to be $\mathscr{F}_{t}$ adapted. In addition, let $x \wedge y=\min \{x, y\}$ below.
Following the same formulation of Zhao et al. [6], the surplus process of an insurer is described by the following jump-diffusion model:

$$
\mathrm{d} R(t)=c d t+\sigma \mathrm{d} W(t)-\mathrm{d} C(t),
$$

where $c$ and $\sigma$ are positive constants, $W(t)$ is a standard Brownian motion, and $\sigma W(t)$ describes the uncertainty associated with the surplus of the insurer at time $t$. Assume $C(t)=\sum_{i=1}^{N(t)} Z_{i}$ which represents the cumulative claims until time $t$, where $N(t)$ is a homogeneous Poisson process with intensity $\lambda$ and the claim sizes $\left\{Z_{i}, i \geq 1\right\}$ are independent and identically distributed positive random variables with common distribution $F(z)$. Denote the mean value $E\left[Z_{i}\right]=$ $\mu_{\infty}$ and

$$
D:=\sup \{z: F(z) \leq 1\}<+\infty .
$$

Suppose that $F(0)=0,0<F(z)<1$ if $z \in(0, D)$ and $F(z)=$ 1 if $z \geq D$. The premium according to the expected value principle is $c=(1+\eta) \lambda \mu_{\infty}$, where $\eta>0$ is the safety loading of the insurer.

According to the theory of Poisson random measure (referring to Øksendal and Sulem [17]), we can rewrite the compound Poisson process $C(t)=\sum_{i=1}^{N(t)} Z_{i} \cdot \gamma$ denotes the Poisson random measure; then

$$
\begin{gathered}
\sum_{i=1}^{N(t)} Z_{i}=\int_{0}^{t} \int_{R^{+}} z \gamma(\mathrm{d} z, \mathrm{~d} u), \\
C^{a}(t)=\sum_{i=1}^{N(t)} Z_{i}=\int_{0}^{t} \int_{R^{+}}(z \wedge a) \gamma(\mathrm{d} z, \mathrm{~d} u) .
\end{gathered}
$$

The compensator $v$ of the random measure $\gamma$ is

$$
\nu(\mathrm{d} z, \mathrm{~d} t)=\lambda \mathrm{d} F(z) \mathrm{d} t,
$$

so the compensated Poisson random measure of $\gamma$ is

$$
\widetilde{\gamma}(\mathrm{d} z, \mathrm{~d} t)=\gamma(\mathrm{d} z, \mathrm{~d} t)-\lambda \mathrm{d} F(z) \mathrm{d} t .
$$

Insurance company can purchase excess-of-loss reinsurance to reduce the risk. Supposing the insurer's (fixed) retention level denoted by $a$, the corresponding reserve process is

$$
\mathrm{d} R(t)=c^{(a)} d t+\sigma \mathrm{d} W(t)-\mathrm{d} C^{(a)}(t)
$$

and the reinsurer's premium rate is

$$
\begin{aligned}
c^{(a)} & =(1+\eta) \lambda \mu_{\infty}-(1+\theta) \lambda\left\{\mu_{\infty}-E\left[Z_{i} \wedge a\right]\right\} \\
& =(\eta-\theta) \lambda \mu_{\infty}+(1+\theta) \lambda E\left[Z_{i} \wedge a\right], \\
E\left[Z_{i} \wedge a\right] & =\int_{0}^{a} z \mathrm{~d} F(z)+\int_{a}^{\infty} a \mathrm{~d} F(z) \\
& =\int_{0}^{a}(1-F(z)) \mathrm{d} z \triangleq \int_{0}^{a} \overline{F(z)} \mathrm{d} z
\end{aligned}
$$


and $C^{(a)}=\sum_{i=1}^{N(t)}\left(Z_{i} \wedge a\right)$ satisfying

$$
e^{\int_{0}^{t} e^{-r s} \mathrm{~d} C^{(a)}(s)}<\infty, \quad \forall t<\infty .
$$

In particular, if the retention level $a=D$, then

$$
c^{(a)}=(1+\eta) \lambda \mu_{\infty}=c^{(D)}=c .
$$

Without loss of generality, we always assume the reinsurance is not cheap; that is, $\theta>\eta$ and $\theta$ denotes the safety loading of the reinsurer.

Moreover, the insurer also can invest in a financial market consisting of one risk-free asset and one risky asset. The price process $S_{0}(t)$ of the risk-free asset is given by

$$
\mathrm{d} S_{0}(t)=r S_{0}(t) \mathrm{d} t
$$

where $r>0$ is the risk-free interest rate. The price process $S(t)$ of the risky asset is described by the constant elasticity of variance $(\mathrm{CEV})$ model:

$$
\mathrm{d} S(t)=S(t)\left[\mu \mathrm{d} t+k S^{\beta}(t) \mathrm{d} W_{1}(t)\right]
$$

where $\mu$ and $k$ are positive constants, $\mu>r$ is the expected instantaneous return rate of the risky asset, $k S^{\beta}(t)$ is the instantaneous volatility, $\beta$ is the elasticity parameter, and $W_{1}(t)$ is a standard Brownian motion. When $\beta=0$, a CEV model degenerates into a GBM. In this paper, we assume the standard Brownian motion $W_{1}(t)$ is independent of $W(t)$.

The control $\alpha=\{(a(t), \pi(t)): t \in[0, T]\}$ is a two-dimensional $\{\mathscr{F}(t)\}$ adapted stochastic process, where $a(t) \in[0, D]$ is the retention level of the excess-of-loss at time $t$, in which $a(t) \equiv D$ means "no reinsurance" and $a(t) \equiv 0$ means "full reinsurance," and $\pi(t)$ represents the proportion invested in the risky asset. Here, short-selling is not allowed; that is, $\pi(t) \geq 0$. The amount invested in the risk-free asset is $X(t)(1-$ $\pi(t))$, where $X(t)$ is the wealth process for the insurer under the strategy $\alpha$ and the dynamics of $X(t)$ is given by

$$
\begin{aligned}
& \mathrm{d} X(t)=[(\eta-\theta) \lambda \mu_{\infty}+(1+\theta) \lambda \int_{0}^{a} \overline{F(x)} \mathrm{d} x \\
&+\mu \pi(t) X(t)+r(1-\pi(t)) X(t)] \mathrm{d} t \\
&+\sigma \mathrm{d} W(t)+\pi(t) X(t) k S^{\beta}(t) \mathrm{d} W_{1}(t)-\mathrm{d} C^{(a)}(t) \\
& X(0)=x_{0} .
\end{aligned}
$$

A strategy $\alpha$ is said to be admissible, if $\forall t \in$ $[0, T],(a(t), \pi(t))$ is $\mathscr{F}(t)$ progressively measurable, and $E\left[\int_{0}^{\infty} \pi^{2}(t) X^{2}(t) S^{2 \beta}(t)\right]<\infty, a(t) \in[0, D]$, and $\pi(t) \in$ $[0,+\infty)$. The set of all admissible strategies denoted by $\Lambda$ and (12) has a unique (strong) solution. Suppose the insurer has a utility function $U(x)$ which is strictly concave and continuously differentiable on $(-\infty,+\infty)$ and aims to maximize the expected utility of his/her terminal wealth; that is,

$$
\max _{\alpha \in \Lambda} E[U(X(T))]
$$

\section{Main Results}

In this section, we solve the excess-of-loss reinsurance and investment problem with independence of two Brownian motions $W(t)$ and $W_{1}(t)$. By maximizing the expected utility of terminal wealth, the optimal strategy and value function are given. At the beginning, let us give the following exponential utility function of an risk aversion insurer:

$$
U(x)=-\frac{1}{q} e^{-q x}, \quad q>0 .
$$

This utility function has a constant absolute risk aversion parameter $q$ and is the only utility function under the principle of "zero utility" giving a fair premium that is independent of the level of reserves of insurers. For an admissible strategy $\alpha=(a(t), \pi(t))$, we define the value function as

$$
H^{\alpha}(t, x, s)=E[U(X(T)) \mid X(t)=x, S(t)=s]
$$

and the optimal value function is

$$
H(t, x, s)=\sup _{\alpha \in \Lambda} H^{\alpha}(t, x, s)
$$

with the boundary condition

$$
H(T, x, s)=-\frac{1}{q} e^{-q x} .
$$

The objective of the insurer is to find an optimal strategy $\alpha^{*}=\left(a^{*}(t), \pi^{*}(t)\right)$ such that $H(t, x, s)=H^{\alpha^{*}}(t, x, s)$, where $a^{*}(t)$ is called the optimal reinsurance strategy and $\pi^{*}(t)$ is called the optimal investment strategy.

For any $H^{\alpha} \in C^{1,2,2}\left([0, T] \times R_{+} \times R_{+}\right)$, define the generator

$$
\begin{aligned}
& \mathscr{A} H^{\alpha}(t, x, s) \\
& =H_{t}^{\alpha}+\left[(\eta-\theta) \lambda \mu_{\infty}+r x\right] H_{x}^{\alpha} \\
& +\mu s H_{s}^{\alpha}+\frac{1}{2} \sigma^{2} H_{x x}^{\alpha}+\frac{1}{2} k^{2} s^{2 \beta+2} H_{s s}^{\alpha} \\
& +\left\{(\mu-r) \pi x H_{x}^{\alpha}+\frac{1}{2} \pi^{2} x^{2} k^{2} s^{2 \beta} H_{x x}^{\alpha}+k^{2} \pi x s^{2 \beta+1} H_{x s}^{\alpha}\right\} \\
& +\left\{H_{x}^{\alpha}(1+\theta) \lambda \int_{0}^{a} \overline{F(x)} \mathrm{d} x\right. \\
& \left.\quad+\lambda E\left[H^{\alpha}\left(t, x-\left(Z_{i} \wedge a\right), s\right)-H^{\alpha}(t, x, s)\right]\right\},
\end{aligned}
$$

where $H_{t}^{\alpha}, H_{x}^{\alpha}, H_{s}^{\alpha}, H_{x x}^{\alpha}$, and $H_{s s}^{\alpha}$ denote the corresponding first- and second-order partial derivatives of $H^{\alpha}(t, x, s)$ with respect to (w.r.t.) the corresponding variables, respectively.

Applying the classical tools of stochastic optimal control, we can derive the following Hamilton-Jacobi-Bellman (HJB) equation for problem (16):

$$
\sup _{\alpha \in \Lambda}\left\{\mathscr{A} H^{\alpha}(t, x, s)\right\}=0
$$

with the boundary condition (17). 
Standard results (e.g., Fleming and Soner [18]) tell us (19) admits the unique strong solution, which, together with the fact that the value function is twice-continuously differentiable, gives the following theorem.

Theorem 1. For the optimal excess-of-loss reinsurance and investment problem with jump-diffusion risk process under the CEV model, a solution to HJB equation (19) with boundary condition (17) is given by $V(t, x, s)$ and the corresponding maximizer is given by $\alpha^{*}=\left(a^{*}, \pi^{*}\right)$ in feedback form, where one has the following.

(1) If $D \geq \ln (1+\theta) / q$, the optimal retention level on the whole interval $[0, T]$ always is

$$
a_{*}^{0}(t)=\frac{\ln (1+\theta)}{q} e^{-r(T-t)}
$$

and the optimal investment strategy is given by

$$
\pi^{*}(t)=\left[\frac{\mu-r}{x q k^{2}}-\frac{(\mu-r)^{2}\left(e^{2 r \beta(t-T)}-1\right)}{2 q x r k^{2}}\right] e^{-r(T-t)} s^{-2 \beta} .
$$

The optimal value function is

$V(t, x, s)$

$$
\begin{aligned}
=-\frac{1}{q} \exp \{- & \\
\times & {\left[x e^{r(T-t)}+\frac{(\eta-\theta) \lambda \mu_{\infty} e^{r(T-t)}}{r}-\frac{\sigma^{2} q e^{2 r(T-t)}}{4 r}\right.} \\
& +\int_{t}^{T}\left(e^{r(T-y)}(1+\theta) \lambda\right. \\
& \left.\quad \times \int_{0}^{(\ln (1+\theta) / q) e^{-r(T-y)}} \overline{F(x)} \mathrm{d} x\right) \mathrm{d} y \\
& -\frac{(\eta-\theta) \lambda \mu_{\infty}}{r}+\frac{\sigma^{2} q}{4 r} \\
+ & \frac{e^{-2 r \beta T}\left(-e^{2 r \beta t}+e^{2 r \beta T}\right)(\mu-r)}{4 q r \beta k^{2}} \int_{0}^{(\ln (1+\theta) / q) e^{-r(T-y)}} e^{q x e^{r(T-y)}} \\
+ & \left(e^{-2 r \beta T}(1+2 \beta) \mathrm{d} y\right. \\
& \times\left(e^{2 r \beta t}+e^{2 r \beta T}(-1+2 r(T-t) \beta)\right) \\
& \left.\left.\left.\times(\mu-r)^{2}\right) \times\left(8 q \beta r^{2}\right)^{-1}\right]\right\} .
\end{aligned}
$$

(2) If $D<\ln (1+\theta) / q$, the optimal retention level is

$$
a^{*}(t)= \begin{cases}\frac{\ln (1+\theta)}{q} e^{-r(T-t)}, & t \in\left[0, t^{0}\right), \\ D, & t \in\left[t^{0}, T\right] .\end{cases}
$$

And the optimal investment strategy is given by

$$
\pi^{*}(t)=\left[\frac{\mu-r}{x q k^{2}}-\frac{(\mu-r)^{2}\left(e^{2 r \beta(t-T)}-1\right)}{2 q x r k^{2}}\right] e^{-r(T-t)} s^{-2 \beta} .
$$

The optimal value function is given by

$V(t, x, s)$

$$
\begin{aligned}
& =-\frac{1}{q} \exp \left\{-q\left[x e^{r(T-t)}+f^{*}(t)+g^{*}(t, s)\right]\right\} \\
& = \begin{cases}-\frac{1}{q} \exp \left\{-q\left[x e^{r(T-t)}+\widetilde{g(t, s)}+\widetilde{f(t)}\right]\right\}, & t \in\left[0, t^{0}\right), \\
-\frac{1}{q} \exp \left\{-q\left[x e^{r(T-t)}+\widehat{g(t, s)}+\widehat{f(t)}\right]\right\}, & t \in\left[t^{0}, T\right],\end{cases}
\end{aligned}
$$

$\widetilde{g(t, s)}$

$$
\begin{aligned}
= & {\left[\left(-\frac{(\mu-r)^{2} e^{-2 r T \beta}}{4 q r k^{2} \beta}\right) e^{2 \beta r t}+\frac{(\mu-r)^{2}}{4 q k^{2} \beta r}\right] s^{-2 \beta} } \\
& +\left(\frac{(\mu-r)^{2} e^{-2 r T \beta}}{4 q r k^{2} \beta}\right) \frac{k^{2}(2 \beta+1)}{2 r} e^{2 \beta r t} \\
& -\frac{(2 \beta+1)}{q r}(\mu-r)^{2}+(2 \beta+1)(\mu-r)^{2} \\
& \times\left(\frac{4+T-t^{0}}{4 q r}-\frac{1}{8 q r^{2} \beta}\right), \quad t \in\left[0, t^{0}\right),
\end{aligned}
$$

$\widehat{g(t, s)}$

$$
\begin{aligned}
= & \frac{\left(1-e^{2 r \beta(t-T)}\right)(\mu-r)^{2}}{4 q k^{2} r \beta} s^{-2 \beta} \\
& +\frac{(1+2 \beta)\left[e^{2 r \beta(t-T)}-1+2 r(T-t) \beta\right](\mu-r)^{2}}{8 q r^{2} \beta}, \\
t \in\left[t^{0}, T\right], &
\end{aligned}
$$

$\widehat{f(t)}$

$$
\begin{aligned}
= & \frac{(1+\eta) \lambda \mu_{\infty} e^{r(T-t)}}{r}-\frac{\sigma^{2} q e^{2 r(T-t)}}{4 r} \\
& -\int_{t}^{T}\left[\frac{\lambda}{q} \int_{0}^{D} e^{q x e^{r(T-\tau)}} \mathrm{d} F(x)\right] \mathrm{d} \tau \\
& -\frac{\lambda(t-T)}{q}-\frac{(1+\eta) \lambda \mu_{\infty}}{r}+\frac{\sigma^{2} q}{4 r}, \quad t \in\left[t^{0}, T\right],
\end{aligned}
$$




$$
\begin{aligned}
\widetilde{f(t)} & \\
= & \frac{(\eta-\theta) \lambda \mu_{\infty}}{r} e^{r(T-t)}-\frac{\sigma^{2} q}{4 r} e^{2 r(T-t)} \\
& -\int_{0}^{t}\left[(1+\theta) \lambda e^{r(T-\tau)} \int_{0}^{(\ln (1+\theta) / q) e^{-r(T-\tau)}} \overline{F(x)} \mathrm{d} x\right] \mathrm{d} \tau \\
& +\lambda \int_{0}^{t}\left[e^{r(T-\tau)} \int_{0}^{(\ln (1+\theta) / q) e^{-r(T-\tau)}} e^{q x e^{r(T-\tau)}} \overline{F(x)} \mathrm{d} x\right] \mathrm{d} \tau \\
& -\frac{(\eta-\theta) \lambda \mu_{\infty}}{r} e^{r\left(T-t^{0}\right)} \\
& +(1+\theta) \lambda \int_{0}^{t^{0}}\left[e^{r(T-\tau)} \int_{0}^{(\ln (1+\theta) / q) e^{-r(T-\tau)}} \overline{F(x)} \mathrm{d} x\right] \mathrm{d} \tau \\
& -\lambda \int_{0}^{t^{0}}\left[e^{r(T-\tau)} \int_{0}^{(\ln (1+\theta) / q) e^{-r(T-\tau)}} e^{q x e^{r(T-\tau)}} \overline{F(x)} \mathrm{d} x\right] \mathrm{d} \tau \\
& +\frac{(1+\eta) \lambda \mu_{\infty}}{r} e^{r\left(T-t^{0}\right)} \\
& -\frac{\lambda}{q} \int_{t^{0}}^{T}\left[\int_{0}^{D} e^{\left.q x e^{r(T-\tau)} \mathrm{d} F(x)\right] \mathrm{d} \tau}\right. \\
& -\frac{\lambda\left(t^{0}-T\right)}{q}-\frac{(1+\eta) \lambda \mu_{\infty}}{r}+\frac{\sigma^{2} q}{4 r}, \quad t \in\left[0, t^{0}\right) .
\end{aligned}
$$

\section{Proof. See Appendix A.}

Remark 2. From Theorem 1, we find that the optimal investment strategy is a function of $(\mu-r), q, x, k s^{\beta}, r$, and $t$, which fully reflects the influence of various factors on the investment strategy. $x$ is the initial wealth, $k s^{\beta}$ represents the volatility of risk asset price, and $\mu-r$ is the profit of risk asset appreciation higher than risk-free interest rate. By simple deformation,

$$
\begin{aligned}
\pi^{*}(t)= & {\left[\frac{\mu-r}{x q k^{2}}-\frac{(\mu-r)^{2}\left(e^{2 r \beta(t-T)}-1\right)}{2 q x r k^{2}}\right] e^{-r(T-t)} s^{-2 \beta} } \\
= & (\mu-r)\left[\frac{1}{q}-\frac{(\mu-r)\left(1-e^{-2 r \beta(T-t)}\right)}{2 q r}\right] \\
& \cdot e^{-r(T-t)} \cdot \frac{1}{x} \cdot \frac{1}{\left(k s^{\beta}\right)^{2}} .
\end{aligned}
$$

Obviously, the optimal investment strategy decreases with respect to the initial wealth $x$ and the volatility of risk asset price $k s^{\beta}$. In particular, if $\beta=0$, then $\pi^{*}(t)=((\mu-r) /$ $\left.x q k^{2}\right) e^{-r(T-t)}$ which is the same as that in Theorem 1 in Gu et al. [5].

A surprising finding is that the optimal reinsurance strategy has nothing to do with the initial wealth and risk asset. However, insurer's safe load $\theta$ and the parameter $q$ in the utility function play an important role in determining reinsurance strategy.
Motivated by the results of Taksar and Zeng [16], Gu et al. [5], and Zhao et al. [6], we obtain the following verification theorem.

Theorem 3 (verification theorem). For the optimal excess-ofloss reinsurance and investment problem with jump-diffusion risk process under the CEV model, the wealth process $X(t)$ is associated with an admissible strategy $(a(t), \pi(t))$. If the solution to HJB equation (19) with boundary condition (17) is given by $V(t, x, s)$ and the parameters $\mu>r>0$ satisfy either of the following conditions:

$$
\begin{aligned}
& \text { (I) } r>(1-1 / \sqrt{6}) \mu ; \\
& \text { (II) } r<(1-1 / \sqrt{6}) \mu ; \quad T \\
& \quad\left(1 / \beta \sqrt{6(\mu-r)^{2}-\mu^{2}}\right) \arctan \left(-\sqrt{6(\mu-r)^{2}-\mu^{2}} / \mu\right)
\end{aligned}
$$

then the optimal value function is $H(t, x, s)=V(t, x, s)$, and the optimal strategy is $\alpha^{*}=\left(a^{*}(t), \pi^{*}(t)\right)$, given in Theorem 1 .

Proof. See Appendix B.

Remark 4. In contrast with Gu et al. [5], they also considered the excess-of-loss with a compound Poisson jump under the CEV model; however, they simplified the compound Poisson jump by diffusion approximation process which leads the problem back to the case without jump.

\section{Numerical Results}

In this section, we analyze the impacts of some parameters on the optimal strategies and the value function. Theoretical analysis and some corresponding numerical examples are given to illustrate the influences of model parameters on the optimal strategy and the optimal value function when $D \geq$ $\ln (1+\theta) / q$. The analysis for the case of $D<\ln (1+\theta) / q$ is similar.

Throughout this section, we always assume $\beta \geq 0$; the claim sizes follow exponential distribution $F(x)=1-e^{-x / m}$, $x>0$, and if $\beta<0$, some of the conclusions will be different. The parameters are given by $m=2, q=0.5, T=10, \sigma=1$, $\mu_{\infty}=0.5, \theta=0.2, r=0.05, \mu=0.12, k=0.2, \lambda=2, x=1$, $\beta=0.5$, and $s=1$.

(1) First, let us pay attention to the expression of the optimal strategy when $\beta \geq 0$ :

$$
\begin{aligned}
\pi^{*}(t)= & {\left[\frac{\mu-r}{x q k^{2}}-\frac{(\mu-r)^{2}\left(e^{2 r \beta(t-T)}-1\right)}{2 q x r k^{2}}\right] e^{-r(T-t)} s^{-2 \beta} } \\
= & {\left[\frac{\mu-r}{q}-\frac{(\mu-r)^{2}\left(e^{2 r \beta(t-T)}-1\right)}{2 q r}\right] } \\
& \cdot e^{-r(T-t)} \cdot \frac{1}{x} \cdot \frac{1}{\left(k s^{\beta}\right)^{2}},
\end{aligned}
$$

where $x$ is the initial wealth and $k s^{\beta}$ represents the volatility of risk asset price. 


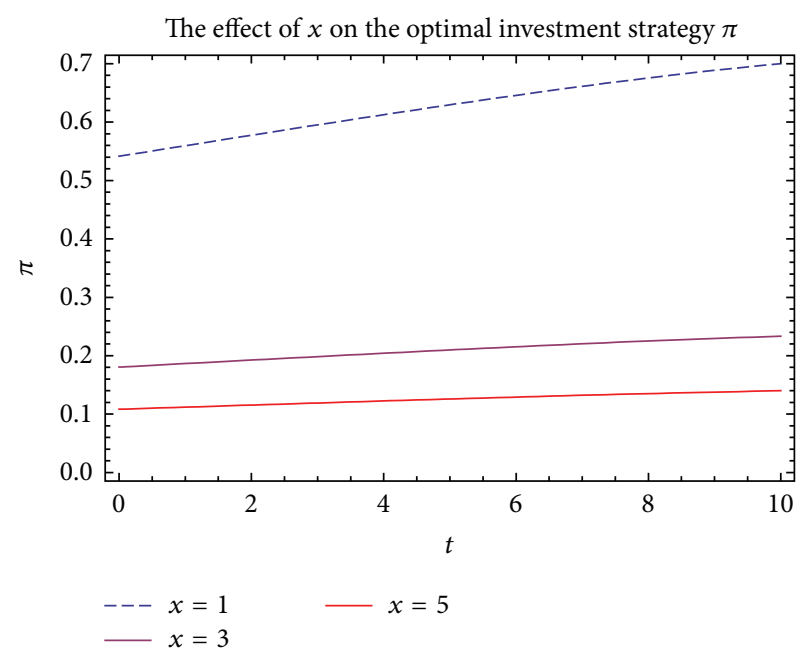

FIGURE 1: The optimal investment strategy $\pi$ decreases with respect to $x$.

Deriving $\pi^{*}$ with respect to $x$, we have

$$
\begin{aligned}
\frac{\partial \pi^{*}}{\partial x}= & \left(e^{r(t-T(1+2 \beta))} s^{-2 \beta}(r-\mu)\right. \\
& \left.\times\left[\left(e^{2 r t \beta}(r-\mu)+e^{2 r T \beta}(r+\mu)\right)\right]\right) \times\left(2 k^{2} q r x^{2}\right)^{-1} \\
= & \left((\mu-r) s^{-2 \beta} e^{r(t-T(1+2 \beta))}\right. \\
& \left.\times\left[r\left(e^{2 r T \beta}+e^{2 r t \beta}\right)+\mu\left(e^{2 r T \beta}-e^{2 r t \beta}\right)\right]\right) \\
& \times\left(2 k^{2} q r x^{2}\right)^{-1}<0,
\end{aligned}
$$

because $\mu>r>0, T>t, s^{-2 \beta}>0, e^{r(t-T(1+2 \beta))}>0$, and $\mu\left(e^{2 r T \beta}-e^{2 r t \beta}\right)>0$.

Remark 5. Obviously, the optimal investment strategy $\pi^{*}$ decreases with respect to the initial wealth $x$, which tells us that, for a risk aversion insurer with the initial fund bigger and bigger, the investment proportion on risk asset must be reduced to make the actual amount invested on risk asset stay at an appropriate level to avoid the potential risk of being unbearable. See Figure 1.

However, the optimal strategy increases with respect to appreciation rate $\mu$ of the risky asset.

Since $T>t, \mu>r>0$ so that $e^{2 r T \beta}>e^{2 r t \beta}$ and $\mu>(\mu-r)$, we have

$$
\frac{\partial \pi^{*}}{\partial \mu}=\frac{e^{r(t-T(1+2 \beta))} s^{-2 \beta}\left[e^{2 r T \beta} \mu-e^{2 r t \beta}(\mu-r)\right]}{k^{2} q r x}>0 .
$$

Figure 2 shows that the optimal strategy increases with respect to the return rate of the risky asset $\mu$, which is obviously established. The higher appreciation rate $\mu$ of risky asset will attract more money invested on the risk asset and force the investment proportion $\pi$ increase. See Figure 2 .

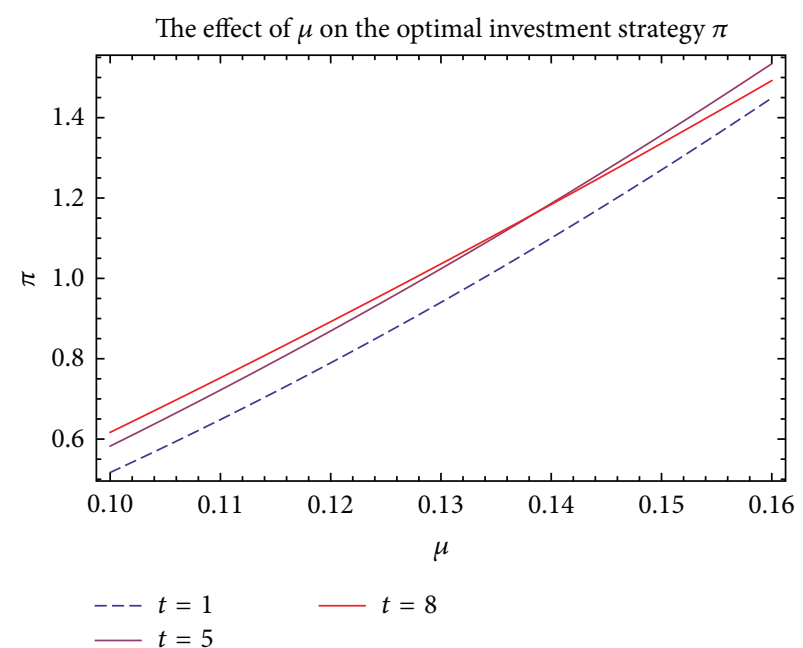

FIgURE 2: The optimal investment strategy increases with the appreciation rate $\mu$ of risky asset.

Proposition 6. Suppose $\beta \geq 0$ is the volatility parameter in CEV model; $\pi^{*}$ is the optimal investment strategy. If $s^{2}>$ $e^{(\mu-r)(T-t)}, s>1, \mu>r>0, T>t$, then the optimal strategy $\pi^{*}$ decreases with respect to $k, \beta$, and s. In fact, the optimal investment strategy decreases with the volatility rate of risk asset price denoted by $k s^{\beta}(t)$.

Proof. The optimal strategy with respect to $\beta$ may raise various possible cases under different conditions:

$$
\begin{aligned}
& \frac{\partial \pi^{*}}{\partial \beta}=-\left(e^{r(t-T(1+2 \beta))} s^{-2 \beta}(r-\mu)\right. \\
& \times\left[e^{2 r t \beta}(r-\mu)(r(t-T)-\ln [s])\right. \\
&\left.\left.-e^{2 r T \beta}(r+\mu) \ln [s]\right]\right) \times\left(k^{2} q r x\right)^{-1} \\
&=\left(e^{r(t-T(1+2 \beta))} s^{-2 \beta}(\mu-r)\right. \\
& \times\left[e^{2 r t \beta}(\mu-r)(r(T-t)+\ln [s])\right. \\
&\left.\left.\quad-e^{2 r T \beta}(r+\mu) \ln [s]\right]\right) \times\left(k^{2} q r x\right)^{-1} .
\end{aligned}
$$

Since $s>1, \mu>r$, and $T>t$, then $\mu-r>0, T-t>0$, $e^{2 r T \beta}>e^{2 r t \beta}>0$, and $\ln [s]>0$.

If $s^{2}>e^{(\mu-r)(T-t)}$, take logarithm on both sides

$$
\ln s^{2}>(\mu-r)(T-t), \quad \text { i.e., } 2 \ln s>(\mu-r)(T-t) .
$$

We have

$$
\begin{aligned}
e^{2 r T \beta} 2 r \ln [s]-e^{2 r t \beta} r(\mu-r)(T-t) & \\
& >e^{2 r t \beta} r(2 \ln [s]-(\mu-r)(T-t))>0,
\end{aligned}
$$


so that

$$
\begin{aligned}
\frac{\partial \pi^{*}}{\partial \beta}= & \left(k^{2} q r x\right)^{-1} e^{r(t-T(1+2 \beta))} s^{-2 \beta}(\mu-r) \\
\times & {\left[e^{2 r t \beta}(\mu-r) \ln [s]\right.} \\
& \quad+e^{2 r t \beta}(\mu-r) r(T-t)-e^{2 r T \beta} \\
& \left.\times(\mu-r) \ln [s]-2 r \ln [s] e^{2 r T \beta}\right] \\
= & \left(k^{2} q r x\right)^{-1} e^{r(t-T(1+2 \beta))} s^{-2 \beta}(\mu-r) \\
\times & -\left(e^{2 r T \beta}-e^{2 r t \beta}\right)(\mu-r) \ln [s] \\
& \left.\quad-\left(e^{2 r T \beta} 2 r \ln [s]-e^{2 r t \beta} r(\mu-r)(T-t)\right)\right]<0,
\end{aligned}
$$

which means that the optimal strategy decreases with respect to $\beta$ (see Figure 3(c)):

$$
\begin{aligned}
\frac{\partial \pi^{*}}{\partial s}= & \left(e^{r(t-T(1+2 \beta))} s^{-2 \beta}(r-\mu)\right. \\
& \left.\times\left[\left(e^{2 r t \beta}(r-\mu)+e^{2 r T \beta}(r+\mu)\right)\right]\right) \times\left(2 k^{2} q r x^{2}\right)^{-1} \\
=- & \left((\mu-r) s^{-2 \beta} e^{r(t-T(1+2 \beta))}\right. \\
& \left.\times\left[r\left(e^{2 r T \beta}+e^{2 r t \beta}\right)+\mu\left(e^{2 r T \beta}-e^{2 r t \beta}\right)\right]\right) \\
\times & \left(2 k^{2} q r x^{2}\right)^{-1}<0,
\end{aligned}
$$

because $\mu-r>0, \beta>0, s^{-2 \beta}>0, e^{r(t-T(1+2 \beta))}>0$, and $\mu\left(e^{2 r T \beta}-e^{2 r t \beta}\right)>0$.

Consider

$$
\frac{\partial \pi^{*}}{\partial k}=-\frac{e^{-r(T-t)} s^{-2 \beta}(\mu-r)\left(\mu+r-e^{-2 r(T-t) \beta}(\mu-r)\right)}{k^{3} q r x}<0 .
$$

Thus the optimal strategy decreases with respect to $s$ and $k$, respectively. See Figures 3(a) and 3(b).

All the calculations above show that the optimal strategy decreases with respect to $k, s$, and $\beta$ simultaneously. So it comes to a conclusion that the optimal investment strategy decreases with the volatility rate $k s^{\beta}(t)$ of risk asset price.

Remark 7. The conclusion of this proposition is very natural. Risk averse investors always adopt a relatively conservative investment strategy to avoid risk. With the increase of risk asset price volatility rate $k s^{\beta}(t)$ which means risk increase, the risk averse insurer must reduce the investment proportion $\pi$ on risk asset to keep the company's risk at an appropriate level. So the optimal investment strategy $\pi^{*}$ decreases with the risk asset price volatility rate $k s^{\beta}(t)$. See Figure 3 .

(2) Next, we are concerned with the expression of the value function for the special case when $\eta=\theta=0$, which makes the value function simplified completely. Without loss of generality, we also suppose $\beta \geq 0$; the case of $\beta<0$ is similar. Hence,

$$
\begin{aligned}
& V(t, x, s) \\
& =-\frac{1}{q} \exp \\
& \quad \times\left\{-q\left[x e^{r(T-t)}-\frac{\sigma^{2} q e^{2 r(T-t)}}{4 r}\right.\right. \\
& \quad+\frac{e^{-2 r \beta T}\left(-e^{2 r \beta t}+e^{2 r \beta T}\right)(\mu-r)^{2}}{4 q r \beta k^{2}} s^{-2 \beta} \\
& \quad+\left(e^{-2 r \beta T}(1+2 \beta)\right. \\
& \left.\quad \times\left(e^{2 r \beta t}+e^{2 r \beta T}(-1+2 r(T-t) \beta)\right)(\mu-r)^{2}\right) \\
& \left.\left.\quad \times\left(8 q \beta r^{2}\right)^{-1}+\frac{\sigma^{2} q}{4 r}\right]\right\} .
\end{aligned}
$$

Proposition 8. $V(t, x, s)$ is the optimal value function under the optimal strategy $\left(a^{*}, \pi^{*}\right)$ and $k$ is the volatility coefficient of risk asset price in CEV model. If $k>0, s>0, \mu>r>0$, and $T>t$, then the optimal value function $V(t, x, s)$ decreases with respect to $k$ and s, respectively.

Proof. If $k>0, s>0, \mu>r>0$, and $T>t$, then

$$
\left(2 k^{3} q r \beta\right)^{-1}\left(e^{2 r T \beta}-e^{2 r t \beta}\right) s^{-2 \beta}(r-\mu)^{2}>0,
$$

so that

$$
\begin{aligned}
\frac{\partial V}{\partial k}=-\left(2 k^{3} q r \beta\right)^{-1}\left(e^{2 r T \beta}-e^{2 r t \beta}\right) s^{-2 \beta}(r-\mu)^{2} \\
\cdot \exp \left\{-2 r T \beta-\frac{1}{8} q\right. \\
\times\left[8 e^{r(-t+T)} x-\frac{2\left(-1+e^{2 r(-t+T)}\right) q \sigma^{2}}{r}\right. \\
-\left(k^{2} q r^{2} \beta\right)^{-1} e^{-2 r T \beta} s^{-2 \beta}(r-\mu)^{2} \\
\times\left[2\left(e^{2 r t \beta}-e^{2 r T \beta}\right) r+k^{2} s^{2 \beta}(1+2 \beta)\right. \\
\left.\left.\times\left(-e^{2 r t \beta}+e^{2 r T \beta}(1+2 r(t-T) \beta)\right)\right]\right\}
\end{aligned}
$$$$
<0,
$$

which means the value function decreases with respect to $k$. See Figure 4(b). 


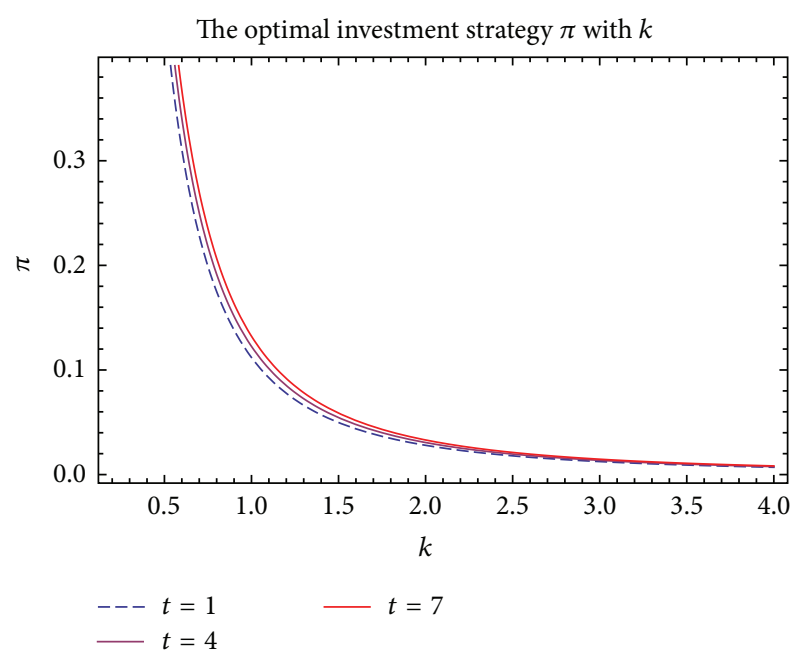

(a)

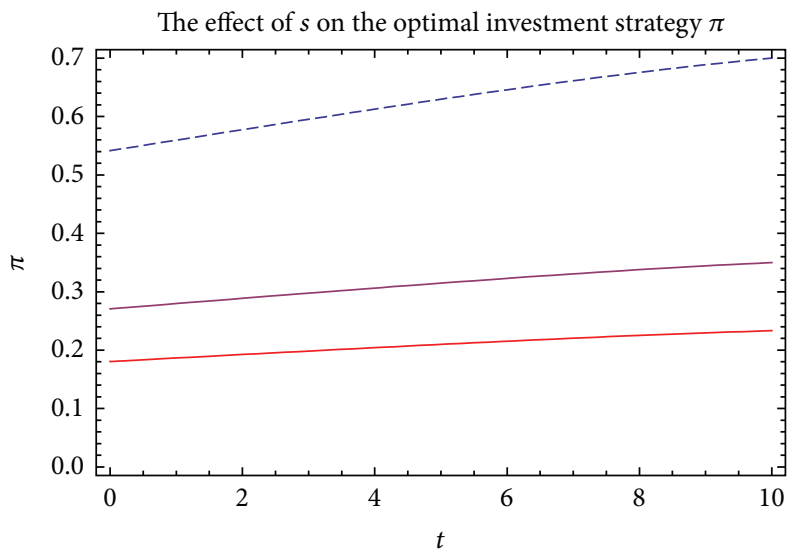

$$
\begin{aligned}
--s & =5 \\
-s & =10
\end{aligned}-s=15
$$

(b)

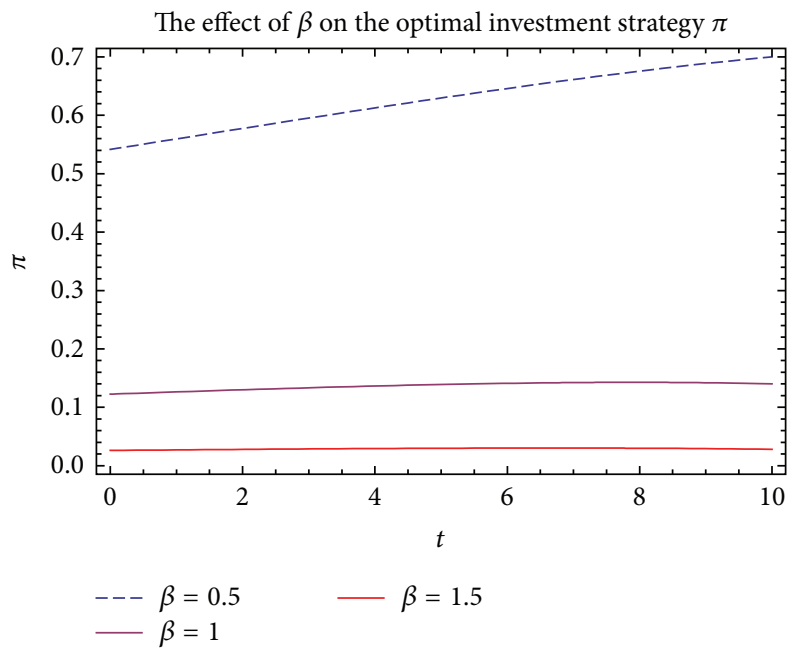

(c)

FIGURE 3: (a) The optimal investment strategy decreases with respect to $k$. (b) The optimal investment strategy $\pi$ decreases with respect to $s$. (c) The optimal investment strategy $\pi$ decreases with respect to $\beta$. 


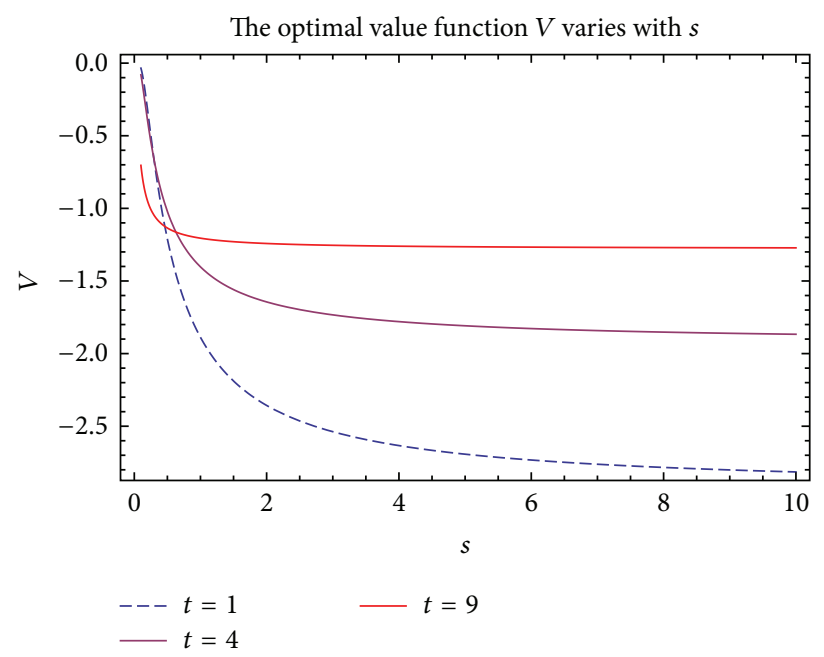

(a)

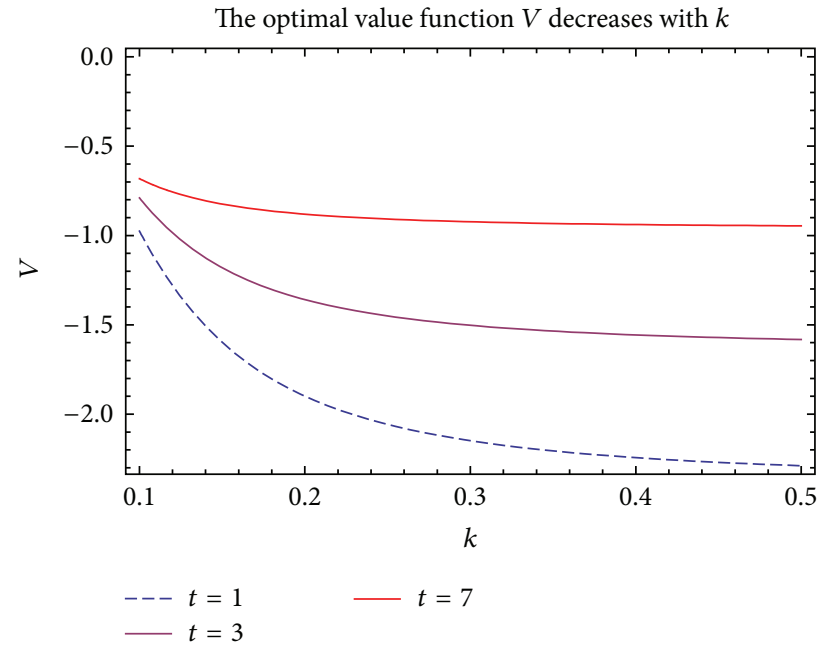

(b)

Figure 4: (a) The optimal value function $V$ decreases with $s$. (b) The value function $V$ decreases with $k$.

Completely similar to the analysis in (1), we also have

$$
\begin{aligned}
& \frac{\partial V}{\partial s}=-\left(2 k^{2} q r\right)^{-1}\left(e^{2 r T \beta}-e^{2 r t \beta}\right) s^{-1-2 \beta}(r-\mu)^{2} \\
& \cdot \exp \left\{-2 r T \beta-\frac{1}{8} q\right. \\
& \times\left[8 e^{r(-t+T)} x-\frac{2\left(-1+e^{2 r(-t+T)}\right) q \sigma^{2}}{r}\right. \\
& -\left(k^{2} q r^{2} \beta\right)^{-1} e^{-2 r T \beta} s^{-2 \beta}(r-\mu)^{2} \\
& \cdot\left[2\left(e^{2 r t \beta}-e^{2 r T \beta}\right) r+k^{2} s^{2 \beta}(1+2 \beta)\right. \\
& \left.\left.\left.\times\left(-e^{2 r t \beta}+e^{2 r T \beta}(1+2 r(t-T) \beta)\right)\right]\right]\right\}
\end{aligned}
$$

$<0$, which shows that the value function decreases with respect to s. See Figure 4(a).

Let

$$
\begin{aligned}
D^{\beta}(t) \triangleq & k^{2} s^{2 \beta} \\
& \times\left(e^{2 r T \beta}\left(1+4 r(T-t) \beta^{2}\right)\right. \\
& \left.\quad-e^{2 r t \beta}(1+2 r(T-t) \beta(1+2 \beta))\right) \\
& +2 e^{2 r t \beta} r(1+2 r(T-t) \beta+2 \beta \ln [s]) \\
& -2 e^{2 r T \beta} r(1+2 \beta \ln [s]) \\
\frac{\partial V}{\partial \beta}= & \left(8 k^{2} q r^{2} \beta^{2}\right)^{-1} s^{-2 \beta}(r-\mu)^{2} \\
& \cdot\left\{k ^ { 2 } s ^ { 2 \beta } \left(e^{2 r T \beta}\left(1+4 r(T-t) \beta^{2}\right)-e^{2 r t \beta}\right.\right. \\
& \times(1+2 r(T-t) \beta(1+2 \beta))) \\
& +2 e^{2 r t \beta} r(1+2 r(T-t) \beta+2 \beta \ln [s]) \\
& \left.-2 e^{2 r T \beta} r(1+2 \beta \ln [s])\right\}
\end{aligned}
$$




$$
\begin{aligned}
\cdot \exp \{- & 2 r T \beta-\frac{1}{8} q \\
\times & {\left[8 e^{r(-t+T)} x-\frac{2\left(-1+e^{2 r(-t+T)}\right) q \sigma^{2}}{r}\right.} \\
& -\left(k^{2} q r^{2} \beta\right)^{-1} e^{-2 r T \beta} s^{-2 \beta}(r-\mu)^{2} \\
& \times\left[2\left(e^{2 r t \beta}-e^{2 r T \beta}\right) r+k^{2} s^{2 \beta}(1+2 \beta)\right. \\
& \times\left(-e^{2 r t \beta}+e^{2 r T \beta}\right. \\
& \times(1+2 r(t-T) \beta))]\} .
\end{aligned}
$$

Obviously, $D^{\beta}(T)=0$. Deriving $D^{\beta}$ defined above with respect to $t$, we have

$$
\begin{aligned}
\frac{\partial D^{\beta}}{\partial t}=4 r \beta^{2}\{ & -e^{2 r T \beta} k^{2} s^{2 \beta}+2 e^{2 r t \beta} r \ln [s] \\
+e^{2 r t \beta}( & 2 r^{2}(-t+T)+k^{2} s^{2 \beta} \\
& \times(1+r(t-T)(1+2 \beta)))\} .
\end{aligned}
$$

Remark 9. If $t$ is big enough relative to $T$, then $\partial D^{\beta} / \partial t>0$, which tells us that $D^{\beta}(t) \rightarrow 0$ increases as time $t$ closes to $T$. So $D^{\beta}(t)<0$ as $t \rightarrow T$. The sign of $\partial V / \partial \beta$ is the same as $D^{\beta}(t)$; that is, $\partial V / \partial \beta<0$ when $t$ is large enough relative to $T$, which means the value function decreases with respect to $\beta$ when $t$ is large enough. Else if $t$ is small enough relative to $T$, the value function may increase with respect to $\beta$.

In conclusion, the volatility $k s^{\beta}$ of risk asset has a significant negative influence on the expected utility of insurance company; through varying parameters $k, s$, and $\beta$ some approximate rule can be found.

Proposition 10. $V(t, x, s)$ is the optimal value function under the optimal strategy $\left(a^{*}, \pi^{*}\right)$ and $\mu$ is the expected return of risk asset price in CEV model. If $\beta \geq 0, s>0, \mu>r>0$, and $T>t$, then the optimal value function $V(t, x, s)$ increases with respect to the appreciation rate of risk asset $\mu$.

Proof. Letting

$$
\begin{aligned}
D^{\mu}(t) \triangleq & {\left[k^{2} s^{2 \beta}(1+2 \beta)-2 r\right]\left(e^{2 r T \beta}-e^{2 r t \beta}\right) } \\
& -2 r(T-t) \beta k^{2} s^{2 \beta}(1+2 \beta) e^{2 r T \beta},
\end{aligned}
$$

obviously, $D^{\mu}(T)=0$ and

$$
\begin{aligned}
\frac{\partial D^{\mu}}{\partial t}= & {\left[k^{2} s^{2 \beta}(1+2 \beta)-2 r\right]\left(-2 r \beta e^{2 r t \beta}\right) } \\
& +2 r \beta k^{2} s^{2 \beta}(1+2 \beta) e^{2 r T \beta}
\end{aligned}
$$

$$
\begin{aligned}
= & -2 r \beta e^{2 r t \beta} k^{2} s^{2 \beta}(1+2 \beta) \\
& +4 r^{2} \beta e^{2 r t \beta}+2 r \beta k^{2} s^{2 \beta}(1+2 \beta) e^{2 r T \beta} \\
= & 2 r \beta k^{2} s^{2 \beta}(1+2 \beta)\left(e^{2 r T \beta}-e^{2 r t \beta}\right) \\
& +4 r^{2} \beta e^{2 r t \beta}>0,
\end{aligned}
$$

which shows that $D^{\mu}(t)$ increases to $D^{\mu}(T)=0$ with respect to time $t$. Thus

$$
\begin{aligned}
& {\left[k^{2} s^{2 \beta}(1+2 \beta)-2 r\right]\left(e^{2 r T \beta}-e^{2 r t \beta}\right)} \\
& \quad-2 r(T-t) \beta k^{2} s^{2 \beta}(1+2 \beta) e^{2 r T \beta} \leq 0 .
\end{aligned}
$$

So

$$
\begin{aligned}
\frac{\partial V}{\partial \mu}= & -\left(4 k^{2} q r^{2} \beta\right)^{-1} s^{-2 \beta}(\mu-r) \\
& \cdot\left\{\left[k^{2} s^{2 \beta}(1+2 \beta)-2 r\right]\left(e^{2 r T \beta}-e^{2 r t \beta}\right)\right. \\
& \left.-2 r(T-t) \beta k^{2} s^{2 \beta}(1+2 \beta) e^{2 r T \beta}\right\} \\
\cdot & \exp \left\{\begin{array}{l}
-2 r T \beta-\frac{1}{8} q \\
\times \\
\quad\left[8 e^{r(-t+T)} x-\frac{2\left(-1+e^{2 r(-t+T)}\right) q \sigma^{2}}{r}\right. \\
\quad\left(k^{2} q r^{2} \beta\right)^{-1} e^{-2 r T \beta} s^{-2 \beta}(r-\mu)^{2} \\
\quad\left[2\left(e^{2 r t \beta}-e^{2 r T \beta}\right) r+k^{2} s^{2 \beta}(1+2 \beta)\right. \\
\left.\times\left(-e^{2 r t \beta}+e^{2 r T \beta}(1+2 r(t-T) \beta)\right)\right]
\end{array}\right]
\end{aligned}
$$$$
\geq 0 \text {. }
$$

The value function $V$ increases with $\mu$ referring to Figure 5, which shows that the higher appreciation rate of risk asset will lead to relatively more greater expected wealth utility. As a matter of fact, appreciation rate always is the main driving factor to make the wealth increase. Of course, the insurer's utility maximization can be better realized for a larger appreciation rate.

\section{Conclusion}

In this paper, we describe the dynamics of the risky assets' prices with the CEV model, under which the optimal excessof-loss reinsurance and investment problem with jumpdiffusion risk process is investigated by maximizing the insurer's exponential utility of terminal wealth. Applying 


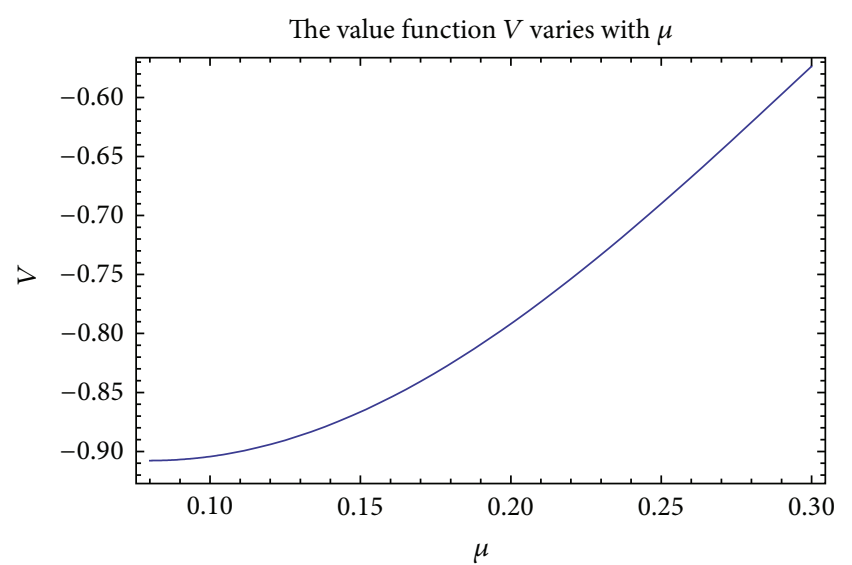

FIGURE 5: The value function $V$ varies with $\mu$.

stochastic control of jump diffusion, a Hamilton-JacobiBellman (HJB) equation associated with a compound Poisson jump risk process is established. The explicit solution for the exponential utility function is given. In the last part of the text, some numerical examples are given to illustrate the effects of model parameters on the optimal strategy and the optimal value function. Meanwhile, we give some propositions and remarks which enriched the innovation of the paper.

\section{Appendices}

\section{A. Proof of Theorem 1}

This appendix collects the proofs of the main results stated in Section 3. Other necessary lemmas are also presented and proved in this appendix.

Proof. Differentiating (19) with respect to $\pi$ gives the optimal investment policy

$$
\pi^{*}=-\frac{(\mu-r) H_{x}^{\alpha}+k^{2} s^{2 \beta+1} H_{x s}^{\alpha}}{x k^{2} s^{2 \beta} H_{x x}^{\alpha}} .
$$

Putting (A.1) into (19), after simplification, we have

$$
\begin{aligned}
H_{t}^{\alpha}+ & {\left[(\eta-\theta) \lambda \mu_{\infty}+r x\right] H_{x}^{\alpha}+\mu s H_{s}^{\alpha} } \\
& +\frac{1}{2} \sigma^{2} H_{x x}^{\alpha}+\frac{1}{2} k^{2} s^{2 \beta+2} H_{s s}^{\alpha} \\
& -\frac{\left[(\mu-r) H_{x}^{\alpha}+k^{2} s^{2 \beta+1} H_{x s}^{\alpha}\right]^{2}}{2 k^{2} s^{2 \beta} H_{x x}^{\alpha}} \\
& +\max _{a}\left\{H_{x}^{\alpha}(1+\theta) \lambda \int_{0}^{a} \overline{F(x)} \mathrm{d} x\right. \\
& \left.+\lambda E\left[H^{\alpha}\left(t, x-\left(Z_{i} \wedge a\right), s\right)-H^{\alpha}(t, x, s)\right]\right\}
\end{aligned}
$$$$
=0 \text {. }
$$

That is,

$$
\begin{aligned}
H_{t}^{\alpha}+ & {\left[(\eta-\theta) \lambda \mu_{\infty}+r x\right] H_{x}^{\alpha}+\mu s H_{s}^{\alpha}+\frac{1}{2} \sigma^{2} H_{x x}^{\alpha} } \\
& +\frac{1}{2} k^{2} s^{2 \beta+2} H_{s s}^{\alpha}-\frac{(\mu-r)^{2}\left(H_{x}^{\alpha}\right)^{2}}{2 k^{2} s^{2 \beta} H_{x x}^{\alpha}} \\
& -\frac{k^{2} s^{2 \beta+2}\left(H_{x s}^{\alpha}\right)^{2}}{2 H_{x x}^{\alpha}}-\frac{(\mu-r) s H_{x}^{\alpha} H_{x s}^{\alpha}}{H_{x x}^{\alpha}} \\
& +\max _{a}\left\{H_{x}^{\alpha}(1+\theta) \lambda \int_{0}^{a} \overline{F(x)} \mathrm{d} x\right. \\
& \left.+\lambda E\left[H^{\alpha}\left(t, x-\left(Z_{i} \wedge a\right), s\right)-H^{\alpha}(t, x, s)\right]\right\}
\end{aligned}
$$$$
=0 .
$$

We try to conjecture (A.2) having a solution $V(t, x, s)$ of this form

$$
\begin{aligned}
& V(t, x, s)=-\frac{1}{q} e^{\left\{-q\left[x e^{r(T-t)}+f(t)+g(t, s)\right]\right\}}, \quad t \in[0, T] \\
& f(T)=0 \\
& g(T, s)=0 \\
& V(T, x, s)=-\frac{1}{q} e^{-q x} .
\end{aligned}
$$

Differentiating the solution (A.4) with respect to the corresponding variables,

$$
\begin{gathered}
V_{t}=V(-q)\left[-r x e^{r(T-t)}+f^{\prime}(t)+g_{t}\right], \\
V_{x}=V(-q) e^{r(T-t)}, \quad V_{x x}=V(-q)^{2} e^{2 r(T-t)}, \\
V_{s}=V(-q) g_{s}, \quad V_{s s}=V\left[(-q)^{2} g_{s}^{2}+(-q) g_{s s}\right], \\
V_{x s}=V(-q)^{2} e^{r(T-t)} g_{s}, \\
E\left[H^{\alpha}\left(t, x-\left(Z_{i} \wedge a\right), s\right)-H^{\alpha}(t, x, s)\right] \\
=V q e^{r(T-t)} \int_{0}^{a} e^{q x e^{r(T-t)}} \overline{F(x)} \mathrm{d} x .
\end{gathered}
$$

Plugging the above derivatives (A.5) into (A.1) gives

$$
\pi^{*}=\frac{\mu-r}{x q k^{2} s^{2 \beta} e^{r(T-t)}}-\frac{s g_{s}}{x e^{r(T-t)}}
$$


and substituting the above derivatives into (A.2) yields

$$
\begin{aligned}
f^{\prime}(t)+g_{t}+(\eta-\theta) \lambda \mu_{\infty} e^{r(T-t)}+r s g_{s} \\
-\frac{1}{2} \sigma^{2} q e^{2 r(T-t)}+\frac{1}{2} k^{2} s^{2 \beta+2} g_{s s}+\frac{(\mu-r)^{2}}{2 q k^{2}} s^{-2 \beta} \\
+\max _{a}\left\{(1+\theta) \lambda e^{r(T-t)} \int_{0}^{a} \overline{F(x)} \mathrm{d} x\right. \\
\left.\quad-\lambda e^{r(T-t)} \int_{0}^{a} e^{q x e^{r(T-t)}} \overline{F(x)} \mathrm{d} x\right\}=0 .
\end{aligned}
$$

Differentiating with respect to $a$ in (A.7), we find that the minimizer $a_{*}^{0}(t)$ satisfies

$$
\left[(1+\theta)-e^{q a^{0}(t) e^{r(T-t)}}\right] \overline{F\left(a^{0}(t)\right)}=0 .
$$

If $a^{0}(t) \in[0, D)$, then $0<\overline{F\left(a^{0}(t)\right)} \leq 1$ and $(1+\theta)-$ $e^{q a^{0}(t) e^{r(T-t)}}=0$, which gives

$$
a_{*}^{0}(t)=\frac{\ln (1+\theta)}{q} e^{-r(T-t)} .
$$

To ensure that $0 \leq a_{*}^{0}(t)<D$, that is, $0 \leq(\ln (1+\theta) /$ $q) e^{-r(T-t)}<D$, it must be ensured that

$$
t<T+\frac{1}{r} \ln \left[\frac{q D}{\ln (1+\theta)}\right] \triangleq t^{0},
$$

which leads to two different cases.

Case 1. If $D \geq(\ln (1+\theta) / q)$, then $t^{0} \geq T$ and

$$
a_{*}^{0}(t)=\frac{\ln (1+\theta)}{q} e^{-r(T-t)}
$$

always is optimal retention level on the whole interval $[0, T]$.

Inserting (A.5) into (A.1), the optimal investment policy is given by

$$
\pi^{*}(t)=\frac{\mu-r}{x q k^{2} s^{2 \beta} e^{r(T-t)}}-\frac{s g_{s}}{x e^{r(T-t)}}
$$

and $a^{*}=a^{0}(t)=(\ln (1+\theta) / q) e^{-r(T-t)}$. The corresponding HJB equation (A.7) becomes

$$
\begin{aligned}
f^{\prime}(t) & +g_{t}+r s g_{s}+(\eta-\theta) \lambda \mu_{\infty} e^{r(T-t)} \\
& -\frac{1}{2} \sigma^{2} q e^{2 r(T-t)}+\frac{1}{2} k^{2} s^{2 \beta+2} g_{s s} \\
& +\frac{(\mu-r)^{2}}{2 q k^{2} s^{2 \beta}}+e^{r(T-t)}(1+\theta) \lambda \\
& \times \int_{0}^{(\ln (1+\theta) / q) e^{-r(T-t)}} \overline{F(x)} \mathrm{d} x-\lambda e^{r(T-t)} \\
& \times \int_{0}^{(\ln (1+\theta) / q) e^{-r(T-t)}} e^{q x e^{r(T-t)}} \overline{F(x)} \mathrm{d} x=0 .
\end{aligned}
$$

To solve (A.13), we decompose (A.13) into two equations:

$$
\begin{aligned}
& f^{\prime}(t)+(\eta-\theta) \lambda \mu_{\infty} e^{r(T-t)}-\frac{1}{2} \sigma^{2} q e^{2 r(T-t)} \\
&+e^{r(T-t)}(1+\theta) \lambda \int_{0}^{(\ln (1+\theta) / q) e^{-r(T-t)}} \overline{F(x)} \mathrm{d} x \\
&-\lambda e^{r(T-t)} \int_{0}^{(\ln (1+\theta) / q) e^{-r(T-t)}} e^{q x e^{r(T-t)}} \overline{F(x)} \mathrm{d} x \\
&= 0, \\
& g_{t}+r s g_{s}+\frac{1}{2} k^{2} s^{2 \beta+2} g_{s s}+\frac{(\mu-r)^{2}}{2 q k^{2} s^{2 \beta}}=0 .
\end{aligned}
$$

By simple transposition and integration for (A.14) with $f(T)=0$, we have

$$
\begin{aligned}
f(t)= & \frac{(\eta-\theta) \lambda \mu_{\infty} e^{r(T-t)}}{r}-\frac{\sigma^{2} q e^{2 r(T-t)}}{4 r} \\
& +\int_{t}^{T}\left(e^{r(T-y)}(1+\theta) \lambda \int_{0}^{(\ln (1+\theta) / q) e^{-r(T-y)}} \overline{F(x)} \mathrm{d} x\right) \mathrm{d} y \\
& -\int_{t}^{T}\left(\lambda e^{r(T-y)} \int_{0}^{(\ln (1+\theta) / q) e^{-r(T-y)}} e^{q x e^{r(T-y)}} \overline{F(x)} \mathrm{d} x\right) \mathrm{d} y \\
& -\frac{(\eta-\theta) \lambda \mu_{\infty}}{r}+\frac{\sigma^{2} q}{4 r} .
\end{aligned}
$$

To solve (A.20), we conjecture a solution with the following form with $A(T)=0$ and $B(T)=0$ :

$$
g(t, s)=A(t) s^{-2 \beta}+B(t) .
$$

Differentiating (A.17) with respect to the corresponding variables, respectively,

$$
\begin{gathered}
g_{t}=A^{\prime}(t) s^{-2 \beta}+B^{\prime}(t), \quad g_{s}=-2 \beta s^{-2 \beta-1} A(t), \\
g_{s s}=2 \beta(2 \beta+1) s^{-2 \beta-2} A(t) .
\end{gathered}
$$

Substituting the above derivatives into (A.20) yields

$$
\begin{aligned}
A^{\prime}(t) & s^{-2 \beta}+B^{\prime}(t)-2 r \beta s^{-2 \beta} A(t) \\
& +k^{2} \beta(2 \beta+1) A(t)+\frac{(\mu-r)^{2}}{2 q k^{2}} s^{-2 \beta}=0 .
\end{aligned}
$$

To solve (A.20), we decompose (A.20) into two equations:

$$
\begin{gathered}
{\left[A^{\prime}(t)-2 r \beta A(t)+\frac{(\mu-r)^{2}}{2 q k^{2}}\right] s^{-2 \beta}=0,} \\
B^{\prime}(t)+k^{2} \beta(2 \beta+1) A(t)=0 .
\end{gathered}
$$

Obviously, $s^{-2 \beta}>0$, (A.20) is equivalent to

$$
A^{\prime}(t)-2 r \beta A(t)+\frac{(\mu-r)^{2}}{2 q k^{2}}=0 .
$$


Equation (A.22) with $A(T)=0$ is easy to be solved and the solution is

$$
A(t)=\frac{e^{-2 r \beta T}\left(-e^{2 r \beta t}+e^{2 r \beta T}\right)(\mu-r)^{2}}{4 q r \beta k^{2}} .
$$

Inserting (A.23) into (A.21) with $B(T)=0$, we have

$$
\begin{aligned}
B(t)=( & e^{-2 r \beta T}(1+2 \beta) \\
& \times\left(e^{2 r \beta t}+e^{2 r \beta T}(-1+2 r(T-t) \beta)\right) \\
& \left.\times(\mu-r)^{2}\right) \times\left(8 q \beta r^{2}\right)^{-1} .
\end{aligned}
$$

Substituting (A.23) and (A.24) back into (A.17), we obtain

$$
\begin{aligned}
g(t, s)= & \frac{e^{-2 r \beta T}\left(-e^{2 r \beta t}+e^{2 r \beta T}\right)(\mu-r)^{2}}{4 q r \beta k^{2}} s^{-2 \beta} \\
& +\left(e^{-2 r \beta T}(1+2 \beta)\right. \\
& \times\left(e^{2 r \beta t}+e^{2 r \beta T}(-1+2 r(T-t) \beta)\right) \\
& \left.\times(\mu-r)^{2}\right) \times\left(8 q \beta r^{2}\right)^{-1} .
\end{aligned}
$$

Substituting (A.16) and (A.25) back into (A.4), the optimal value function is given by

$$
\begin{aligned}
& V(t, x, s) \\
& =-\frac{1}{q} \exp \\
& \times\{-q \\
& \times\left[x e^{r(T-t)}+\frac{(\eta-\theta) \lambda \mu_{\infty} e^{r(T-t)}}{r}-\frac{\sigma^{2} q e^{2 r(T-t)}}{4 r}\right. \\
& +\int_{t}^{T}\left(e^{r(T-y)}(1+\theta) \lambda\right. \\
& \left.\times \int_{0}^{(\ln (1+\theta) / q) e^{-r(T-y)}} \overline{F(x)} \mathrm{d} x\right) \mathrm{d} y \\
& -\int_{t}^{T}\left(\lambda e^{r(T-y)}\right. \\
& \left.\times \int_{0}^{(\ln (1+\theta) / q) e^{-r(T-y)}} e^{q x e^{r(T-y)}} \overline{F(x)} \mathrm{d} x\right) \mathrm{d} y \\
& -\frac{(\eta-\theta) \lambda \mu_{\infty}}{r}+\frac{\sigma^{2} q}{4 r}
\end{aligned}
$$

$$
\begin{aligned}
& +\left(e^{-2 r \beta T}(1+2 \beta)\right. \\
& \times\left(e^{2 r \beta t}+e^{2 r \beta T}(-1+2 r(T-t) \beta)\right) \\
& \left.\times(\mu-r)^{2}\right) \times\left(8 q \beta r^{2}\right)^{-1} \\
& \left.\left.+\frac{e^{-2 r \beta T}\left(-e^{2 r \beta t}+e^{2 r \beta T}\right)(\mu-r)^{2}}{4 q r \beta k^{2}} s^{-2 \beta}\right]\right\},
\end{aligned}
$$$$
t \in[0, T] \text {. }
$$

Case 2. If $D<\ln (1+\theta) / q$, then $t^{0}<T$; the whole interval $[0, T]$ is divided into two parts, $\left[0, t^{0}\right)$ and $\left[t^{0}, T\right] \cdot a^{0}(t)=$ $(\ln (1+\theta) / q) e^{-r(T-t)}$ is the optimal retention level only on $\left[0, t^{0}\right)$; however, $a^{0}(t) \geq D$ on $\left[t^{0}, T\right]$; we can only take $D$ as the retention level naturally. Therefore, the optimal retention level is denoted by

$$
a^{*}(t)= \begin{cases}\frac{\ln (1+\theta)}{q} e^{-r(T-t)}, & t \in\left[0, t^{0}\right), \\ D, & t \in\left[t^{0}, T\right] .\end{cases}
$$

The solving process depends on two different time intervals corresponding to the different optimal retention level, respectively.

(1) For

$$
a^{*}(t)=D, \quad t \in\left[t^{0}, T\right]
$$

the corresponding HJB equation is

$$
\begin{aligned}
& H_{t}^{\alpha}+\left[(1+\eta) \lambda \mu_{\infty}+r x\right] H_{x}^{\alpha}+\mu s H_{s}^{\alpha} \\
& +\frac{1}{2} \sigma^{2} H_{x x}^{\alpha}+\frac{1}{2} k^{2} s^{2 \beta+2} H_{s s}^{\alpha} \\
& +\max _{\pi}\left\{(\mu-r) \pi x H_{x}^{\alpha}+\frac{1}{2} \pi^{2} x^{2} k^{2} s^{2 \beta} H_{x x}^{\alpha}\right. \\
& \left.\quad+k^{2} \pi x s^{2 \beta+1} H_{x s}^{\alpha}\right\} \\
& +\lambda E\left[H^{\alpha}\left(t, x-\left(Z_{i} \wedge D\right), s\right)-H^{\alpha}(t, x, s)\right]=0 .
\end{aligned}
$$

The first-order maximizing condition for the optimal investment strategy is

$$
\pi^{*}(t)=-\frac{(\mu-r) H_{x}^{\alpha}+k^{2} s^{2 \beta+1} H_{x s}^{\alpha}}{x k^{2} s^{2 \beta} H_{x x}^{\alpha}} .
$$


Inserting (A.30) into (A.29) and simplifying, the HJB equation becomes

$$
\begin{aligned}
H_{t}^{\alpha}+ & {\left[(1+\eta) \lambda \mu_{\infty}+r x\right] H_{x}^{\alpha}+\mu s H_{s}^{\alpha} } \\
& +\frac{1}{2} \sigma^{2} H_{x x}^{\alpha}+\frac{1}{2} k^{2} s^{2 \beta+2} H_{s s}^{\alpha} \\
& -\frac{\left[(\mu-r) H_{x}^{\alpha}+k^{2} s^{2 \beta+1} H_{x s}^{\alpha}\right]^{2}}{2 k^{2} s^{2 \beta} H_{x x}^{\alpha}} \\
& +\lambda E\left[H^{\alpha}\left(t, x-\left(Z_{i} \wedge D\right), s\right)-H^{\alpha}(t, x, s)\right]=0 .
\end{aligned}
$$

We conjecture (A.31) having a solution $V(t, x, s)$ as follows:

$$
\begin{gathered}
V(t, x, s)=-\frac{1}{q} \exp \left\{-q\left[x e^{r(T-t)}+\widehat{f(t)}+\widehat{g(t, s)}\right]\right\}, \\
t \in\left[t^{0}, T\right], \\
V(T, x, s)=U(x), \\
\widehat{f(T)}=0, \\
\widehat{g(T, s)}=0 .
\end{gathered}
$$

Differentiating the conjecture (A.32) with respect to the corresponding variables,

$$
\begin{gathered}
V_{t}=V(-q)\left[-r x e^{r(T-t)}+\widehat{f^{\prime}(t)}+\widehat{g_{t}}\right] \\
V_{x}=V(-q) e^{r(T-t)}, \quad V_{x x}=V(-q)^{2} e^{2 r(T-t)}, \\
V_{s}=V(-q) \widehat{g_{s}}, \quad V_{s s}=V\left[(-q)^{2} \widehat{g}_{s}^{2}+(-q) \widehat{g_{s s}}\right], \\
V_{x s}=V(-q)^{2} e^{r(T-t)} \widehat{g_{s}}, \\
E\left[H^{\alpha}\left(t, x-\left(Z_{i} \wedge D\right), s\right)-H^{\alpha}(t, x, s)\right] \\
=V \int_{0}^{D} e^{q x e^{r(T-t)}} \mathrm{d} F(x)-V .
\end{gathered}
$$

Substituting the above derivatives (A.33) into (A.31) and (A.30), after simplifying it gives

$$
\begin{gathered}
\widehat{\pi^{*}}(t)=\frac{(\mu-r)}{q k^{2} x s^{2} \beta e^{r(T-t)}}-\frac{s \widehat{s}}{x e^{r(T-t)}}, \\
\widehat{f^{\prime}(t)}+\widehat{g_{t}}+(1+\eta) \lambda \mu_{\infty} e^{r(T-t)}-\frac{1}{2} \sigma^{2} q e^{2 r(T-t)} \\
+r s \widehat{g_{s}}+\frac{1}{2} k^{2} s^{2 \beta+2} \widehat{g}_{s s}+\frac{(\mu-r)^{2}}{2 q k^{2}} s^{-2 \beta} \\
-\frac{\lambda}{q} \int_{0}^{D} e^{q x e^{r(T-t)}} \mathrm{d} F(x)+\frac{\lambda}{q}=0 .
\end{gathered}
$$

Decompose (A.35) into two equations

$$
\begin{gathered}
\widehat{f^{\prime}(t)}+(1+\eta) \lambda \mu_{\infty} e^{r(T-t)}-\frac{1}{2} \sigma^{2} q e^{2 r(T-t)} \\
-\frac{\lambda}{q} \int_{0}^{D} e^{q x e^{r(T-t)}} \mathrm{d} F(x)+\frac{\lambda}{q}=0, \\
\widehat{g_{t}}+r s \widehat{g}_{s}+\frac{1}{2} k^{2} s^{2 \beta+2} \widehat{g}_{s s}+\frac{(\mu-r)^{2}}{2 q k^{2}} s^{-2 \beta}=0 .
\end{gathered}
$$

By transposition and integration for (A.36) with $\widehat{f(T)}=0$,

$$
\begin{aligned}
\widehat{f(t)}= & \frac{(1+\eta) \lambda \mu_{\infty} e^{r(T-t)}}{r}-\frac{\sigma^{2} q e^{2 r(T-t)}}{4 r} \\
& -\int_{t}^{T}\left[\frac{\lambda}{q} \int_{0}^{D} e^{q x e^{r(T-\tau)}} \mathrm{d} F(x)\right] \mathrm{d} \tau \\
& -\frac{\lambda(t-T)}{q}-\frac{(1+\eta) \lambda \mu_{\infty}}{r}+\frac{\sigma^{2} q}{4 r} .
\end{aligned}
$$

For (A.37), we try to find a solution as follows:

$$
\widehat{g(t, s)}=\widehat{A(t)} s^{-2 \beta}+\widehat{B(t)}
$$

satisfying $\widehat{A(T)}=0$ and $\widehat{B(T)}=0$. Equation (A.37) turns into

$$
\begin{gathered}
\widehat{A^{\prime}(t)} s^{-2 \beta}+\widehat{B^{\prime}(t)}-2 r \beta \widehat{A(t)} s^{-2 \beta}+k^{2} \beta(2 \beta+1) \widehat{A(t)} \\
+\frac{(\mu-r)^{2}}{2 q k^{2}} s^{-2 \beta}=0 .
\end{gathered}
$$

Decompose (A.40) into two equations

$$
\begin{gathered}
{\left[\widehat{A^{\prime}(t)}-2 r \beta \widehat{A(t)}+\frac{(\mu-r)^{2}}{2 q k^{2}}\right] s^{-2 \beta}=0,} \\
\widehat{B^{\prime}(t)}+k^{2} \beta(2 \beta+1) \widehat{A(t)}=0 .
\end{gathered}
$$

Solving the above simple ordinary differential equations, we get

$$
\begin{gathered}
\widehat{A(t)}=\frac{\left(1-e^{2 r \beta(t-T)}\right)(\mu-r)^{2}}{4 q k^{2} r \beta}, \\
\widehat{B(t)}=\frac{(1+2 \beta)\left[e^{2 r \beta(t-T)}-1+2 r(T-t) \beta\right](\mu-r)^{2}}{8 q r^{2} \beta} .
\end{gathered}
$$

So

$$
\begin{aligned}
& \widehat{g(t, s)}= \frac{\left(1-e^{2 r \beta(t-T)}\right)(\mu-r)^{2}}{4 q k^{2} r \beta} s^{-2 \beta} \\
&+\frac{(1+2 \beta)\left[e^{2 r \beta(t-T)}-1+2 r(T-t) \beta\right](\mu-r)^{2}}{8 q r^{2} \beta}, \\
& \widehat{\pi^{*}}(t)=\frac{2 r(\mu-r)+(\mu-r)^{2}\left(1-e^{2 r \beta(t-T)}\right)}{2 q k^{2} r x s^{2 \beta} e^{r(T-t)}} .
\end{aligned}
$$


(2) For

$$
a^{*}(t)=\frac{\ln (1+\theta)}{q} e^{-r(T-t)}, \quad t \in\left[0, t^{0}\right),
$$

the corresponding HJB equation is the same as (A.29), which corresponds to $\left[0, t^{0}\right)$ having a solution $V(t, x, s)$ as follows:

$$
\begin{gathered}
V(t, x, s)=-\frac{1}{q} \exp \left\{-q\left[x e^{r(T-t)}+\widetilde{f(t)}+\widetilde{g(t, s)}\right]\right\}, t \in\left[0, t^{0}\right], \\
\widetilde{f\left(t^{0}\right)}=\widehat{f\left(t^{0}\right)}, \\
\widetilde{g\left(t^{0}, s\right)}=\widehat{g\left(t^{0}, s\right) .}
\end{gathered}
$$

After the same calculation as (1), we obtain the following results:

$$
\begin{aligned}
\widetilde{f^{\prime}(t)} & +\widetilde{g_{t}}+(\eta-\theta) \lambda \mu_{\infty} e^{r(T-t)}-\frac{1}{2} \sigma^{2} q e^{2 r(T-t)} \\
& +\frac{1}{2} k^{2} s^{2 \beta+2} \widetilde{g_{s s}}+r s \widetilde{g_{s}}+\frac{(\mu-r)^{2}}{2 q k^{2}} s^{-2 \beta} \\
& +(1+\theta) \lambda e^{r(T-t)} \int_{0}^{(\ln (1+\theta) / q) e^{-r(T-t)}} \overline{F(x)} \mathrm{d} x \\
& -\lambda e^{r(T-t)} \int_{0}^{(\ln (1+\theta) / q) e^{-r(T-t)}} e^{q x e^{r(T-t)}} \overline{F(x)} \mathrm{d} x=0 .
\end{aligned}
$$

Splitting (A.46) into two equations,

$$
\widetilde{g_{t}}+\frac{1}{2} k^{2} s^{2 \beta+2} \widetilde{g_{s s}}+r s \widetilde{g_{s}}+\frac{(\mu-r)^{2}}{2 q k^{2}} s^{-2 \beta}=0
$$

The equation (A.47) is a linear second-order partial differential equation with variable coefficients, which exhibits a unique solution according to the appendix of Badaoui and Fernandez [19] or is ensured by the theorem of Friedman [20]:

$$
\begin{aligned}
\widetilde{f^{\prime}(t)} & +(\eta-\theta) \lambda \mu_{\infty} e^{r(T-t)}-\frac{1}{2} \sigma^{2} q e^{2 r(T-t)} \\
& +(1+\theta) \lambda e^{r(T-t)} \int_{0}^{(\ln (1+\theta) / q) e^{-r(T-t)}} \overline{F(x)} \mathrm{d} x \\
& -\lambda e^{r(T-t)} \int_{0}^{(\ln (1+\theta) / q) e^{-r(T-t)}} e^{q x e^{r(T-t)} \overline{F(x)}} \mathrm{d} x=0 .
\end{aligned}
$$

By transposition and integration to (A.48), the result is given by

$$
\begin{aligned}
\widetilde{f(t)}= & \frac{(\eta-\theta) \lambda \mu_{\infty}}{r} e^{r(T-t)}-\frac{\sigma^{2} q}{4 r} e^{2 r(T-t)} \\
& -\int_{0}^{t}\left[(1+\theta) \lambda e^{r(T-\tau)} \int_{0}^{(\ln (1+\theta) / q) e^{-r(T-\tau)}} \overline{F(x)} \mathrm{d} x\right] \mathrm{d} \tau \\
& +\lambda \int_{0}^{t}\left[e^{r(T-\tau)} \int_{0}^{(\ln (1+\theta) / q) e^{-r(T-\tau)}} e^{q x e^{r(T-\tau)} \overline{F(x)} \mathrm{d} x}\right] \mathrm{d} \tau \\
& +C_{3},
\end{aligned}
$$

where $w$ can be determined by $\widetilde{f\left(t^{0}\right)}=\widehat{f\left(t^{0}\right)}$. Consider

$$
\begin{aligned}
C_{3}= & -\frac{(\eta-\theta) \lambda \mu_{\infty}}{r} e^{r\left(T-t^{0}\right)}+(1+\theta) \lambda \\
& \times \int_{0}^{t^{0}}\left[e^{r(T-\tau)} \int_{0}^{(\ln (1+\theta) / q) e^{-r(T-\tau)}} \overline{F(x)} \mathrm{d} x\right] \mathrm{d} \tau \\
& -\lambda \int_{0}^{t^{0}}\left[e^{r(T-\tau)} \int_{0}^{(\ln (1+\theta) / q) e^{-r(T-\tau)}} e^{q x e^{r(T-\tau)}} \overline{F(x)} \mathrm{d} x\right] \mathrm{d} \tau \\
& +\frac{(1+\eta) \lambda \mu_{\infty}}{r} e^{r\left(T-t^{0}\right)} \\
& -\frac{\lambda}{q} \int_{t^{0}}^{T}\left[\int_{0}^{D} e^{q x e^{r(T-\tau)}} \mathrm{d} F(x)\right] \mathrm{d} \tau \\
& -\frac{\lambda\left(t^{0}-T\right)}{q}-\frac{(1+\eta) \lambda \mu_{\infty}}{r}+\frac{\sigma^{2} q}{4 r} .
\end{aligned}
$$

So

$$
f^{*}(t)= \begin{cases}\widetilde{f(t)}, & t \in\left[0, t^{0}\right), \\ \widehat{f(t)}, & t \in\left[t^{0}, T\right] .\end{cases}
$$

With the same calculations as before, we obtain

$$
\begin{aligned}
\widetilde{g(t, s)}= & {\left[C_{1} e^{2 \beta r t}+\frac{(\mu-r)^{2}}{4 q k^{2} \beta r}\right] s^{-2 \beta}-C_{1} \frac{k^{2}(2 \beta+1)}{2 r} e^{2 \beta r t} } \\
& -\frac{(2 \beta+1)}{q r}(\mu-r)^{2}+C_{2}
\end{aligned}
$$

here, the undetermined constants $C_{1}$ and $C_{2}$ will be determined by using the continuity of $V(t, x, s)$.

By the continuity of $V(t, x, s)$ at $t=t^{0}$, we have

$$
\overline{g\left(t^{0}, s\right)}=\widehat{g\left(t^{0}, s\right)} .
$$


Comparing the items and the coefficients on both sides of (A.53), we obtain the undetermined constants $C_{1}$ and $C_{2}$ :

$$
\begin{aligned}
& C_{1}=-\frac{(\mu-r)^{2} e^{-2 r T \beta}}{4 q r k^{2} \beta}, \\
& C_{2}=(2 \beta+1)(\mu-r)^{2}\left(\frac{4+T-t^{0}}{4 q r}-\frac{1}{8 q r^{2} \beta}\right) .
\end{aligned}
$$

So

$$
g^{*}(t, s)= \begin{cases}\widetilde{g(t, s)}, & t \in\left[0, t^{0}\right), \\ \widetilde{g(t, s)}, & t \in\left[t^{0}, T\right] .\end{cases}
$$

And the optimal value function is

$$
V(t, x, s)=-\frac{1}{q} \exp \left\{-q\left[x e^{r(T-t)}+f^{*}(t)+g^{*}(t, s)\right]\right\} .
$$

\section{B. Proof of Theorem 3}

A new lemma is necessary to prove Theorem 3. For convenience, denote $Q:=[0,+\infty) \times[0,+\infty)$.

Lemma B.1. Take a sequence of bounded open sets $Q_{1}, Q_{2}$, $Q_{3}, \ldots$, with $Q_{i} \subset Q_{i+1} \subset Q, i=1,2, \ldots$ and $Q=\cup_{i} Q_{i}$. Let $\tau_{i}$ denote the exit time of $\left(X^{\alpha^{*}}(t), S(t)\right)$ from $Q_{i}$. If the conditions in Theorem 3 hold, then one has $E\left[V^{2}\left(\tau_{i} \wedge T, X^{\alpha^{*}}\left(\tau_{i} \wedge T\right), S\left(\tau_{i} \wedge\right.\right.\right.$ $T)) \mid X(t)=x, S(t)=s]<\infty$ for $i=1,2, \ldots$.

Proof. According to Øksendal and Sulem [17], we rewrite the compound Poisson process $C(t)=\sum_{i=1}^{N(t)} Z_{i}$ in terms of a Poisson random measure. Suppose $\gamma$ is the Poisson random measure; then

$$
\begin{gathered}
\sum_{i=1}^{N(t)} Z_{i}=\int_{0}^{t} \int_{R^{+}} z \gamma(\mathrm{d} z, \mathrm{~d} u), \\
C^{a}(t)=\sum_{i=1}^{N(t)} Z_{i}=\int_{0}^{t} \int_{R^{+}}(z \wedge a) \gamma(\mathrm{d} z, \mathrm{~d} u) .
\end{gathered}
$$

The compensator $v$ of the random measure $\gamma$ is

$$
\nu(\mathrm{d} z, \mathrm{~d} t)=\lambda \mathrm{d} F(z) \mathrm{d} t
$$

so the compensated Poisson random measure of $C(t)$ is

$$
\tilde{\gamma}(\mathrm{d} z, \mathrm{~d} t)=\gamma(\mathrm{d} z, \mathrm{~d} t)-\lambda \mathrm{d} F(z) \mathrm{d} t .
$$
$\widetilde{V}^{2}(t)$

Set $\widetilde{V}(t)=V\left(t, X^{\alpha^{*}}(t), S(t)\right)$. Applying Itô's formula to

$$
\begin{aligned}
& \mathrm{d} \widetilde{V}^{2}(t) \\
& =2 \widetilde{V}(t)\left\{V_{x} \sigma \mathrm{d} W(t)+k S^{\beta}(t)\right. \\
& \left.\quad \times\left[V_{x} \pi(t) X(t)+V_{s} S(t)\right] \mathrm{d} W_{1}(t)+\mathscr{A} \widetilde{V}(t)\right\} \\
& +\left[V_{x}^{2} \sigma^{2}+V_{x}^{2} \pi^{2} X(t)^{2} k^{2} S^{2 \beta}(t)+V_{s}^{2} k^{2} S^{2 \beta+2}(t)\right. \\
& \left.+2 V_{x} V_{s} \pi(t) X(t) k^{2} S^{2 \beta+1}(t)\right] \mathrm{d} t \\
& +\int_{R^{+}}\left[V^{2}(t, X(t-)+(z \wedge a), S(t))\right. \\
& \quad-V^{2}(t, X(t-), S(t))-2 V(t, X(t-), S(t)) \\
& \left.\cdot V_{x} \cdot(z \wedge a)\right] \lambda \mathrm{d} F(z) \mathrm{d} t \\
& +\int_{R^{+}}\left[V^{2}(t, X(t-)+(z \wedge a), S(t))\right. \\
& \left.\quad-V^{2}(t, X(t-), S(t))\right] \widetilde{\gamma}(\mathrm{d} z, \mathrm{~d} t) .
\end{aligned}
$$

Since $\alpha^{*}$ is the optimal strategy for (19), then $\mathscr{A}^{\alpha^{*}} \widetilde{V}(t)=$ 0 . Plugging the expression of $V_{x}, V_{s}$, and $a *(t)$ into (B.4), we obtain the following equation by simple calculations:

$$
\begin{aligned}
& \frac{\mathrm{d} \widetilde{V}^{2}(t)}{\widetilde{V}^{2}(t)} \\
& =\left\{-2 q \sigma e^{r(T-t)} \mathrm{d} W(t)-\frac{2(\mu-r) s^{-\beta}}{k}\right. \\
& \times\left[1+\frac{\left(1-e^{-2 r \beta(T-t)}\right)(\mu-r)}{2 r}\right] \mathrm{d} W_{1}(t) \\
& \left.+\frac{\left(1-e^{-2 r \beta(T-t)}\right)(\mu-r)^{2} s^{-\beta}}{r k} \mathrm{~d} W_{1}(t)\right\} \\
& +\left\{q^{2} \sigma^{2} e^{2 r(T-t)}\right. \\
& +\frac{(\mu-r)^{2} s^{-2 \beta}}{k^{2}}\left[1+\frac{\left(1-e^{-2 r \beta(T-t)}\right)(\mu-r)}{2 r}\right]^{2} \\
& +\frac{\left(1-e^{-2 r \beta(T-t)}\right)^{2}(\mu-r)^{4} s^{-2 \beta}}{4 r^{2} k^{2}} \\
& -\frac{\left(1-e^{-2 r \beta(T-t)}\right)(\mu-r)^{3} s^{-2 \beta}}{r k^{2}} \\
& \left.\times\left[1+\frac{\left(1-e^{-2 r \beta(T-t)}\right)(\mu-r)}{2 r}\right]\right\} \mathrm{d} t
\end{aligned}
$$




$$
\begin{aligned}
& +\int_{R^{+}}\left[e^{-2 q\left(z \wedge a^{*}\right) e^{r(T-t)}}\right. \\
& \left.+2 q\left(z \wedge a^{*}\right) e^{r(T-t)}-1\right] \lambda \mathrm{d} F(z) \mathrm{d} t \\
& +\int_{R^{+}}\left[e^{-2 q\left(z \wedge a^{*}\right) e^{r(T-t)}}-1\right] \tilde{\gamma}(\mathrm{d} z, \mathrm{~d} t) .
\end{aligned}
$$

The solution of (B.5) is

$$
\begin{aligned}
& \frac{\widetilde{V}^{2}(t)}{\widetilde{V}^{2}(0)} \\
& =\exp \left\{\int_{0}^{t}-2 q \sigma e^{r(T-u)} \mathrm{d} W(u)\right. \\
& -\frac{1}{2} \int_{0}^{t} 4 q^{2} \sigma^{2} e^{2 r(T-u)} \mathrm{d} u \\
& +\int_{0}^{t}-\frac{2(\mu-r) s^{-\beta}}{k} \\
& \times\left[1+\frac{\left(1-e^{-2 r \beta(T-u)}\right)(\mu-r)}{2 r}\right] \mathrm{d} W_{1}(u) \\
& -\frac{1}{2} \int_{0}^{t} \frac{4(\mu-r)^{2} s^{-2 \beta}}{k^{2}} \\
& \times\left[1+\frac{\left(1-e^{-2 r \beta(T-u)}\right)(\mu-r)}{2 r}\right]^{2} \mathrm{~d} u \\
& +\int_{0}^{t} \frac{\left(1-e^{-2 r \beta(T-u)}\right)(\mu-r)^{2} s^{-\beta}}{r k} \mathrm{~d} W_{1}(u) \\
& -\frac{1}{2} \int_{0}^{t} \frac{\left(1-e^{-2 r \beta(T-u)}\right)^{2}(\mu-r)^{4} s^{-2 \beta}}{r^{2} k^{2}} \mathrm{~d} u \\
& +\int_{0}^{t} 3 q^{2} \sigma^{2} e^{2 r(T-u)} \mathrm{d} u \\
& +\int_{0}^{t} \frac{3\left(1-e^{-2 r \beta(T-u)}\right)^{2}(\mu-r)^{4} s^{-2 \beta}}{4 r^{2} k^{2}} \mathrm{~d} u \\
& +\int_{0}^{t} \frac{3(\mu-r)^{2} s^{-2 \beta}}{k^{2}} \\
& \times\left[1+\frac{\left(1-e^{-2 r \beta(T-u)}\right)(\mu-r)}{2 r}\right]^{2} \mathrm{~d} u \\
& +\int_{0}^{t}-\frac{\left(1-e^{-2 r \beta(T-u)}\right)(\mu-r)^{3} s^{-2 \beta}}{r k^{2}} \\
& \times\left[1+\frac{\left(1-e^{-2 r \beta(T-u)}\right)(\mu-r)}{2 r}\right] \mathrm{d} u
\end{aligned}
$$

$$
\begin{aligned}
& +\int_{0}^{t} \int_{R^{+}}\left[e^{-2 q\left(z \wedge a^{*}\right) e^{r(T-u)}}\right. \\
& \left.+2 q\left(z \wedge a^{*}\right) e^{r(T-u)}-1\right] \lambda \mathrm{d} F(z) \mathrm{d} u \\
& \left.+\int_{0}^{t} \int_{R^{+}}\left[e^{-2 q\left(z \wedge a^{*}\right) e^{r(T-u)}}-1\right] \tilde{\gamma}(\mathrm{d} z, \mathrm{~d} u)\right\},
\end{aligned}
$$

where $\left(x_{0}, s_{0}\right)$ is the state at time 0 . Applying Itô's formula to (11), we can derive

$$
\begin{aligned}
d S^{-2 \beta}(t)= & \left(\beta(2 \beta+1) k^{2}-2 \beta r S^{-2 \beta}(t)\right) \mathrm{d} t \\
& -2 \beta k \sqrt{S^{-2 \beta}(t)} \mathrm{d} W_{1}(t) .
\end{aligned}
$$

According to the results of Taksar and Zeng [16] and Gu et al. [5] and other existing literature, $\mu>r>0$, we know that

$$
\begin{aligned}
& \exp \left\{\int_{0}^{t}-2 q \sigma e^{r(T-u)} \mathrm{d} W(u)-\frac{1}{2} \int_{0}^{t} 4 q^{2} \sigma^{2} e^{2 r(T-u)} \mathrm{d} u\right\} \\
& \exp \left\{\int_{0}^{t}-\frac{2(\mu-r) s^{-\beta}}{k}\right. \\
& \times\left[1+\frac{\left(1-e^{-2 r \beta(T-u)}\right)(\mu-r)}{2 r}\right] \mathrm{d} W_{1}(u) \\
& -\frac{1}{2} \int_{0}^{t} \frac{4(\mu-r)^{2} s^{-2 \beta}}{k^{2}} \\
& \left.\times\left[1+\frac{\left(1-e^{-2 r \beta(T-u)}\right)(\mu-r)}{2 r}\right]^{2} \mathrm{~d} u\right\}, \\
& \exp \left\{\int_{0}^{t} \frac{\left(1-e^{-2 r \beta(T-u)}\right)(\mu-r)^{2} s^{-\beta}}{r k} \mathrm{dW}_{1}(u)\right. \\
& \left.-\frac{1}{2} \int_{0}^{t} \frac{\left(1-e^{-2 r \beta(T-u)}\right)^{2}(\mu-r)^{4} s^{-2 \beta}}{r^{2} k^{2}} \mathrm{~d} u\right\}
\end{aligned}
$$

are martingales. Since $z \in[0, D], D=\sup \{z: F(z) \leq 1\}<$ $+\infty$, and

$$
E\left[\int_{0}^{T} \int_{R^{+}}\left[e^{-2 q\left(z \wedge a^{*}\right) e^{r(T-u)}}-1\right]^{2} \lambda \mathrm{d} F(z) \mathrm{d} u\right]<\infty,
$$

then the process

$$
\int_{0}^{t} \int_{R^{+}}\left[e^{-2 q\left(z \wedge a^{*}\right) e^{r(T-u)}}-1\right] \tilde{\gamma}(\mathrm{d} z, \mathrm{~d} u)
$$

is also a martingale.

According to Taksar and Zeng [16],

$$
E\left[\exp \left\{\int_{0}^{t} \frac{3(\mu-r)^{2} s^{-2 \beta}(u)}{k^{2}} \mathrm{~d} u\right\}\right]<\infty
$$


when either of conditions (I) and (II) in Theorem 3 is satisfied. Taking expectation from both sides of (B.6) yields

$$
\begin{aligned}
& E\left[\widetilde{V}^{2}(t)\right] \\
& =\widetilde{V}^{2}(0) E \\
& \times\left[\operatorname { e x p } \left\{\int_{0}^{t} 3 q^{2} \sigma^{2} e^{2 r(T-u)} \mathrm{d} u\right.\right. \\
& +\int_{0}^{t} \frac{3\left(1-e^{-2 r \beta(T-u)}\right)^{2}(\mu-r)^{4} s^{-2 \beta}(u)}{4 r^{2} k^{2}} \mathrm{~d} u \\
& \left.+\int_{0}^{t} \frac{3(\mu-r)^{2} s^{-2 \beta}(u)}{k^{2}}\right] \mathrm{d} u \\
& \quad \times\left[1+\frac{\left(1-e^{-2 r \beta(T-u)}\right)(\mu-r)}{2 r}\right]^{2} \\
& +\int_{0}^{t}-\frac{\left(1-e^{-2 r \beta(T-u)}\right)(\mu-r)^{3} s^{-2 \beta}(u)}{r k^{2}} \\
& \quad \times\left[1+\frac{\left(1-e^{-2 r \beta(T-u)}\right)(\mu-r)}{2 r}\right] \mathrm{d} u \\
& +\int_{0}^{t} \int_{R^{+}}\left[e^{-2 q\left(z \wedge a^{*}\right) e^{r(T-u)}}\right. \\
& \\
& \\
& \left.+2 q\left(z \wedge a^{*}\right) e^{r(T-u)}-1\right] \\
& \times \lambda \mathrm{d} F(z) \mathrm{d} u\} .
\end{aligned}
$$

If $\beta \geq 0$, then $0 \leq\left(1-e^{-2 r \beta(T-u)}\right)<1$. For $\mu>r>0$ and $T>0$ are constants, we have

$$
\begin{aligned}
& \exp \left\{\int_{0}^{t} \frac{3(\mu-r)^{2} s^{-2 \beta}(u)}{k^{2}}\right. \\
& \quad \times\left[1+\frac{\left.\left(1-e^{-2 r \beta(T-u)}\right)(\mu-r)\right]^{2}}{2 r} \mathrm{~d} u\right\} \\
& \quad<\exp \left\{\int_{0}^{t} \frac{3(\mu-r)^{2} s^{-2 \beta}(u)}{k^{2}}\left[1+\frac{(\mu-r)}{2 r}\right]^{2} \mathrm{~d} u\right\} \\
& =\exp \left\{\left[1+\frac{(\mu-r)}{2 r}\right]^{2} \int_{0}^{t} \frac{3(\mu-r)^{2} s^{-2 \beta}(u)}{k^{2}} \mathrm{~d} u\right\},
\end{aligned}
$$

$$
\begin{gathered}
\exp \left\{\int_{0}^{t} \frac{3\left(1-e^{-2 r \beta(T-u)}\right)^{2}(\mu-r)^{4} s^{-2 \beta}(u)}{4 r^{2} k^{2}} \mathrm{~d} u\right\} \\
<\exp \left\{\int_{0}^{t} \frac{3(\mu-r)^{4} s^{-2 \beta}(u)}{4 r^{2} k^{2}} \mathrm{~d} u\right\}, \\
\exp \left\{\int_{0}^{t} \mid-\frac{\left(1-e^{-2 r \beta(T-u)}\right)(\mu-r)^{3} s^{-2 \beta}(u)}{r k^{2}}\right. \\
\left.\times\left[1+\frac{\left(1-e^{-2 r \beta(T-u)}\right)(\mu-r)}{2 r}\right] \mid \mathrm{d} u\right\} \\
<\exp \left\{\int_{0}^{t} \frac{(\mu-r)^{3} s^{-2 \beta}(u)}{r k^{2}}\left[1+\frac{(\mu-r)}{2 r}\right] \mathrm{d} u\right\} .
\end{gathered}
$$

For the case of $\beta<0,\left|1-e^{-2 r \beta(T-u)}\right|<e^{-2 r \beta T}$, which is similar. After simple calculation and analysis, we obtain

$$
E\left[V^{2}\left(t, X^{\alpha^{*}}(t), S(t)\right)\right]=E\left[\widetilde{V}^{2}(t)\right]<\infty .
$$

So $E\left[V^{2}\left(\tau_{i} \wedge T, X^{\alpha^{*}}\left(\tau_{i} \wedge T\right), S\left(\tau_{i} \wedge T\right)\right)\right]<\infty$ for $i=1,2, \ldots$

We use Lemma B.1 to prove the verification Theorem 3 as follows.

Proof. By Itô's formula for Itô-Lévy process, we have

$$
\begin{aligned}
\mathrm{d} V\left(t, X^{\alpha}(t), S(t)\right) & \\
= & \mathscr{A}^{\alpha} V\left(t, X^{\alpha}(t), S(t)\right)+V_{x} \sigma \mathrm{d} W(t) \\
+ & k S^{\beta}(t)\left[V_{x} \pi(t) X(t)+V_{s} S(t)\right] \mathrm{d} W_{1}(t) \\
+ & \int_{R^{+}}[V(t, X(t-)+(z \wedge a), S(t)) \\
& -V(t, X(t-), S(t))] \tilde{\gamma}(\mathrm{d} z, \mathrm{~d} t) .
\end{aligned}
$$

Take a sequence of bounded open sets $Q_{1}, Q_{2}, Q_{3}, \ldots$, with $Q_{i} \subset Q_{i+1} \subset Q, i=1,2, \ldots$. For $(x, s) \in Q_{1}$, let $\tau_{i}$ denote the exit time of $(x, s)$ from $Q_{i}$. Then $\tau_{i} \wedge T \rightarrow T$ when $i \rightarrow \infty$. Integrating both sides of the above equation,

$$
\begin{aligned}
& V\left(\tau_{i} \wedge T, X^{\alpha}\left(\tau_{i} \wedge T\right), S\left(\tau_{i} \wedge T\right)\right) \\
& =V(t, x, s)+\int_{t}^{\tau_{i} \wedge T} \mathscr{A}^{\alpha} V\left(u, X^{\alpha}(u), S(u)\right) \mathrm{d} u \\
& +\int_{t}^{\tau_{i} \wedge T} V_{x} \sigma \mathrm{d} W(u) \\
& +\int_{t}^{\tau_{i} \wedge T} k S^{\beta}(u)\left[V_{x} \pi x+V_{s} S(u)\right] \mathrm{d} W_{1}(u) \\
& +\int_{t}^{\tau_{i} \wedge T} \int_{R^{+}}[V(u, X(u-)+(z \wedge a), S(u)) \\
& \quad-V(u, X(u-), S(u))] \tilde{\gamma}(\mathrm{d} z, \mathrm{~d} u) .
\end{aligned}
$$


Since $\sup _{\alpha \in \Lambda}\left\{\mathscr{A}^{\alpha} V(t, x, s)\right\}=0$, then $\mathscr{A}^{\alpha} V(t, x, s)<0$. The last three terms of (B.16) are square-integrable martingales with zero expectation, taking conditional expectation given $(t, x, s)$ on two sides of the above formula:

$$
\begin{aligned}
& E\left[V\left(\tau_{i} \wedge T, X^{\alpha}\left(\tau_{i} \wedge T\right), S\left(\tau_{i} \wedge T\right)\right)\right. \\
& \left.\mid X^{\alpha}(t)=x, S(t)=s\right] \\
& =V(t, x, s) \\
& \quad+E\left[\int_{t}^{\tau_{i} \wedge T} \mathscr{A}^{\alpha} V\left(u, X^{\alpha}(u), S(u)\right) \mathrm{d} u\right. \\
& \left.\quad \mid X^{\alpha}(t)=x, S(t)=s\right] \\
& \leq V(t, x, s) .
\end{aligned}
$$

From Lemma B.1, $V\left(\tau_{i} \wedge T, X^{\alpha}\left(\tau_{i} \wedge T\right), S\left(\tau_{i} \wedge T\right)\right), i=$ $1,2, \ldots$, are uniformly integrable. Thus we have

$$
\begin{aligned}
& H(t, x, s) \\
& \quad=\sup _{\alpha \in \Lambda} E\left[U(X(T)) \mid X^{\alpha^{*}}(t)=x, S(t)=s\right] \\
& \quad=\lim _{t \rightarrow \infty} E\left[V\left(\tau_{i} \wedge T, X^{\alpha}\left(\tau_{i} \wedge T\right), S\left(\tau_{i} \wedge T\right)\right)\right] \\
& \quad \leq V(t, x, s) .
\end{aligned}
$$

When $\alpha=\alpha^{*}$, the inequality in the above formula becomes an equality; that is, $H(t, x, s)=V(t, x, s)$. The proof is finished.

\section{Conflict of Interests}

The authors declare that they have no conflict of interests regarding the publication of this paper.

\section{Acknowledgments}

This research was supported by the National Natural Science Foundation of China (Grant nos. 11201335 and 11301376) and Humanity and Social Science Foundation of Ministry of Education of China (Grant no. 11YJC910007).

\section{References}

[1] K. Borch, "The safety loading of reinsurance premiums," Scandinavian Actuarial Journal, vol. 1960, no. 3-4, pp. 163-184, 1960.

[2] C. S. Tapiero and D. Zuckerman, "Optimum excess-loss reinsurance: a dynamic framework," Stochastic Processes and Their Applications, vol. 12, no. 1, pp. 85-96, 1982.

[3] G. Taylor, "Reserving consecutive layers of inwards excess-ofloss reinsurance," Insurance: Mathematics \& Economics, vol. 20, no. 3, pp. 225-242, 1997.

[4] Y. Cao and J. Xu, "Proportional and excess-of-loss reinsurance under investment gains," Applied Mathematics and Computation, vol. 217, no. 6, pp. 2546-2550, 2010.
[5] A. Gu, X. Guo, Z. Li, and Y. Zeng, "Optimal control of excessof-loss reinsurance and investment for insurers under a CEV model," Insurance: Mathematics \& Economics, vol. 51, no. 3, pp. 674-684, 2012.

[6] H. Zhao, X. Rong, and Y. Zhao, "Optimal excess-of-loss reinsurance and investment problem for an insurer with jumpdiffusion risk process under the Heston model," Insurance: Mathematics \& Economics, vol. 53, no. 3, pp. 504-514, 2013.

[7] H. L. Yang and L. H. Zhang, "Optimal investment for insurer with jump-diffusion risk process," Insurance: Mathematics \& Economics, vol. 37, no. 3, pp. 615-634, 2005.

[8] B. Li, R. Wu, and M. Song, "A renewal jump-diffusion process with threshold dividend strategy," Journal of Computational and Applied Mathematics, vol. 228, no. 1, pp. 41-55, 2009.

[9] X. Ruan, W. Zhu, J. Hu, and J. Huang, "Optimal portfolio and consumption with habit formation in a jump diffusion market," Applied Mathematics and Computation, vol. 222, pp. 391-401, 2013.

[10] J. C. Cox and S. A. Ross, "The valuation of options for alternative stochastic processes," Journal of Financial Economics, vol. 3, no. 1-2, pp. 145-166, 1976.

[11] Y. L. Hsu, T. I. Lin, and C. F. Lee, "Constant elasticity of variance (CEV) option pricing model: integration and detailed derivation," Mathematics and Computers in Simulation, vol. 79, no. 1, pp. 60-71, 2008.

[12] L. Campi, S. Polbennikov, and A. Sbuelz, "Systematic equitybased credit risk: a CEV model with jump to default," Journal of Economic Dynamics \& Control, vol. 33, no. 1, pp. 93-108, 2009.

[13] J. W. Xiao, S. H. Yin, and C. L. Qin, "Constant elasticity of variance model and analytical strategies for annuity contracts," Applied Mathematics and Mechanics, vol. 27, no. 11, pp. 14991506, 2006.

[14] M. Gu, Y. Yang, S. Li, and J. Zhang, "Constant elasticity of variance model for proportional reinsurance and investment strategies," Insurance: Mathematics \& Economics, vol. 46, no. 3, pp. 580-587, 2010.

[15] X. Lin and Y. Li, "Optimal reinsurance and investment for a jump diffusion risk process under the CEV model," North American Actuarial Journal, vol. 15, no. 3, pp. 417-431, 2011.

[16] M. Taksar and X. D. Zeng, "A general stochastic volatility model and optimal portfolio with explicit solutions," Working Paper, 2009.

[17] B. Øksendal and A. Sulem, Applied Stochastic Control of Jump Diffusions, Springer, Berlin, Germany, 2009.

[18] W. H. Fleming and H. M. Soner, Controlled Markov Processes and Viscosity Solutions, Springer, Berlin, Germany, 1993.

[19] M. Badaoui and B. Fernandez, "An optimal investment strategy with maximal risk aversion and its ruin probability in the presence of stochastic volatility on investments," Insurance: Mathematics and Economics, vol. 53, no. 1, pp. 1-13, 2013.

[20] A. Friedman, Stochastic Differential Equations and Applications, Academic Press, New York, NY, USA, 1975. 


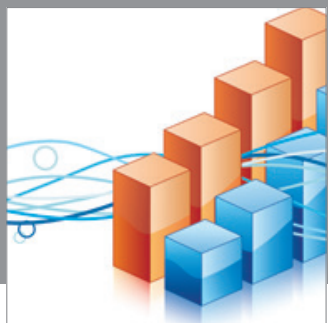

Advances in

Operations Research

mansans

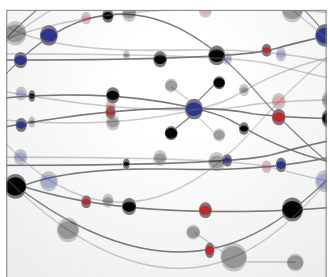

The Scientific World Journal
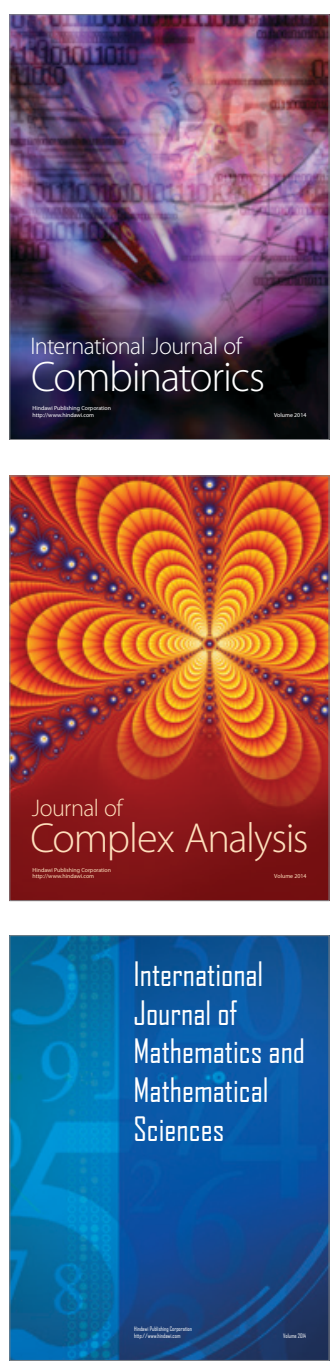
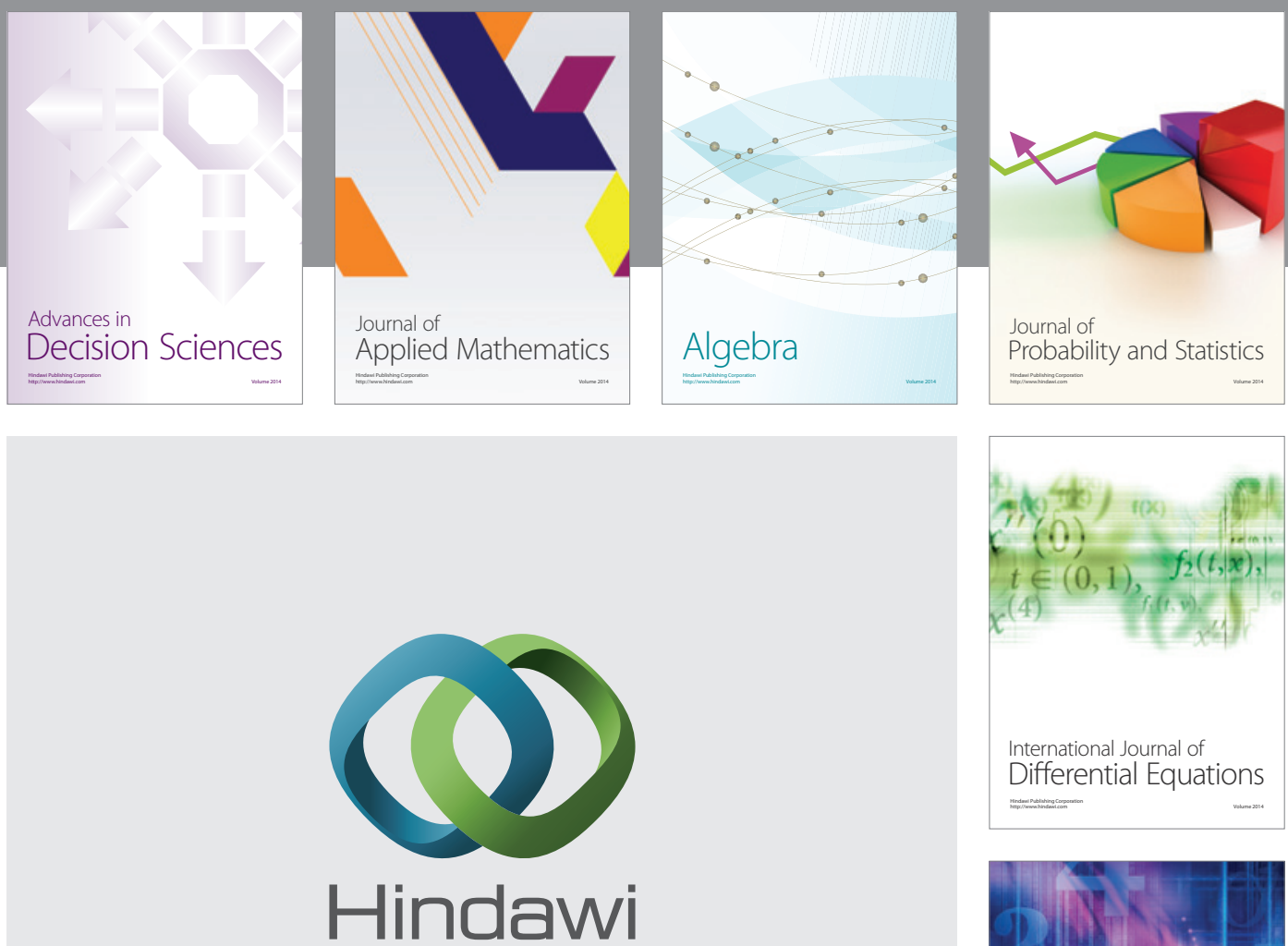

Submit your manuscripts at http://www.hindawi.com
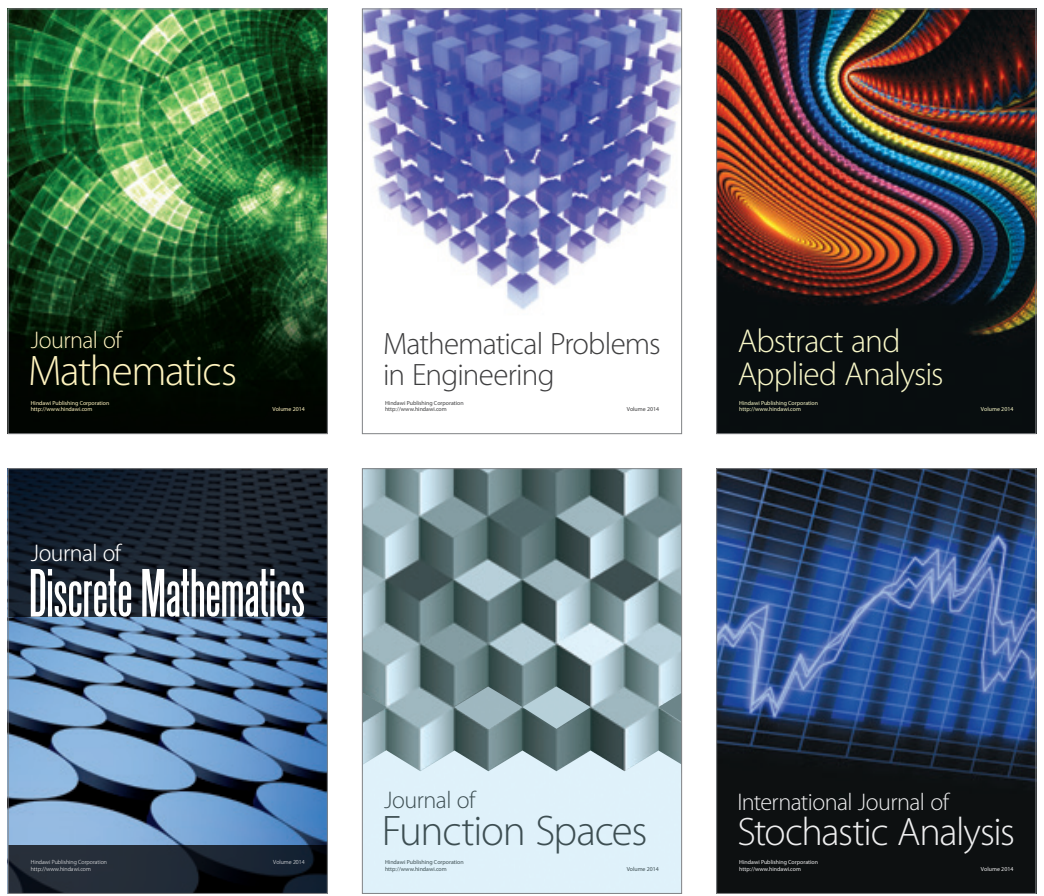

Journal of

Function Spaces

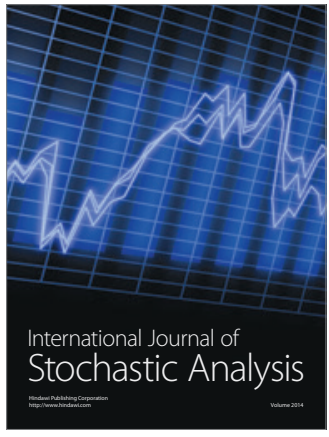

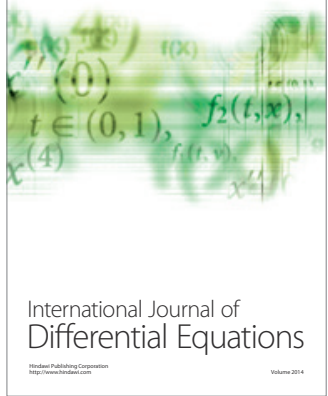
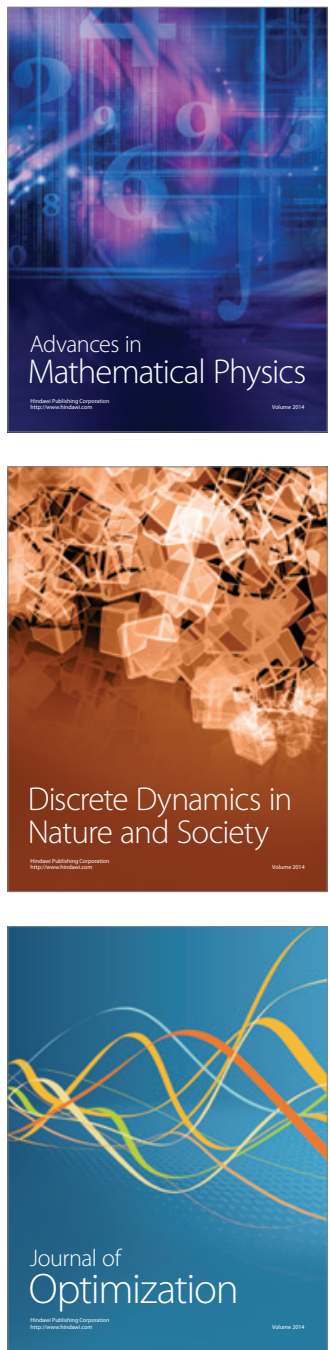